\title{
NMR Metabolite Profiles in Male Meat-Eaters, Fish-Eaters, Vegetarians and Vegans, and Comparison with MS Metabolite Profiles
}

\author{
Julie A. Schmidt ${ }^{1, *(1)}$, Georgina K. Fensom ${ }^{1,2}{ }^{\oplus}$, Sabina Rinaldi ${ }^{3}$, Augustin Scalbert ${ }^{3}\left(\mathbb{D}\right.$, Marc J. Gunter ${ }^{3}$, \\ Michael V. Holmes ${ }^{4,5}$, Timothy J. Key ${ }^{1}$ and Ruth C. Travis ${ }^{1}$
}

1 Cancer Epidemiology Unit, Nuffield Department of Population Health, University of Oxford, Oxford OX3 7LF, UK; georgina.fensom@qeh.ox.ac.uk (G.K.F.); tim.key@ndph.ox.ac.uk (T.J.K.); ruth.travis@ndph.ox.ac.uk (R.C.T.)

2 Department of International Development, University of Oxford, Oxford OX1 3TB, UK

3 Section of Nutrition and Metabolism, International Agency for Research on Cancer, 69372 Lyon, France; rinaldis@iarc.fr (S.R.); scalberta@iarc.fr (A.S.); gunterm@iarc.fr (M.J.G.)

4 Medical Research Council Population Health Research Unit, Nuffield Department of Population Health, University of Oxford, Oxford OX3 7LF, UK; michael.holmes@ndph.ox.ac.uk

5 Clinical Trial Service Unit \& Epidemiological Studies Unit, Nuffield Department of Population Health, University of Oxford, Oxford OX3 7LF, UK

* Correspondence: julie.schmidt@ndph.ox.ac.uk; Tel.: +44-(0)1865-289641

check for updates

Citation: Schmidt, J.A.; Fensom, G.K.; Rinaldi, S.; Scalbert, A.; Gunter, M.J.; Holmes, M.V.; Key, T.J.; Travis, R.C. NMR Metabolite Profiles in Male Meat-Eaters, Fish-Eaters, Vegetarians and Vegans, and Comparison with MS Metabolite Profiles. Metabolites 2021, 11, 121. https://doi.org/ 10.3390/metabo11020121

Academic Editor: Cholsoon Jang

Received: 27 January 2021

Accepted: 14 February 2021

Published: 20 February 2021

Publisher's Note: MDPI stays neutral with regard to jurisdictional claims in published maps and institutional affiliations.

Copyright: (c) 2021 by the authors. Licensee MDPI, Basel, Switzerland. This article is an open access article distributed under the terms and conditions of the Creative Commons Attribution (CC BY) license (https:/ / creativecommons.org/licenses/by/ $4.0 /)$.

\begin{abstract}
Metabolomics may help to elucidate mechanisms underlying diet-disease relationships and identify novel risk factors for disease. To inform the design and interpretation of such research, evidence on diet-metabolite associations and cross-assay comparisons is needed. We aimed to compare nuclear magnetic resonance (NMR) metabolite profiles between meat-eaters, fish-eaters, vegetarians and vegans, and to compare NMR measurements to those from mass spectrometry (MS), clinical chemistry and capillary gas-liquid chromatography (GC). We quantified 207 serum NMR metabolite measures in 286 male participants of the European Prospective Investigation into Cancer and Nutrition (EPIC)-Oxford cohort. Using univariate and multivariate analyses, we found that metabolite profiles varied by diet group, especially for vegans; the main differences compared to meat-eaters were lower levels of docosahexaenoic acid, total n-3 and saturated fatty acids, cholesterol and triglycerides in very-low-density lipoproteins, various lipid factions in highdensity lipoprotein, sphingomyelins, tyrosine and creatinine, and higher levels of linoleic acid, total $n-6$, polyunsaturated fatty acids and alanine. Levels in fish-eaters and vegetarians differed by metabolite measure. Concentrations of 13 metabolites measured using both NMR and MS, clinical chemistry or GC were mostly similar. In summary, vegans' metabolite profiles were markedly different to those of men consuming animal products. The studied metabolomics platforms are complementary, with limited overlap between metabolite classes.
\end{abstract}

Keywords: European Prospective Investigation into Cancer and Nutrition (EPIC)-Oxford; fisheaters; mass spectrometry (MS); meat-eaters; metabolomics; nuclear magnetic resonance (NMR); vegans; vegetarians

\section{Introduction}

Diets with reduced intakes of animal products, including vegetarian and vegan diets, are becoming increasingly popular in the Western world for ethical, environmental and health reasons [1]. Prospective epidemiological evidence suggests that individuals excluding some (e.g., fish-eaters and vegetarian) or all animal products (vegans) from their diets may have different risks of some major non-communicable diseases compared to meat-eaters. This includes lower risks of coronary heart disease [2-4], some cancers [5-8], and diabetes $[9,10]$ but possibly an increased risk of stroke [4] and fractures [11], in some or 
all of the non-meat-eating diet groups. Metabolomics has the potential to reveal underlying mechanisms in such diet-disease relationships [12], because metabolite concentrations reflect dietary, lifestyle, environmental and genetic factors as well as disease states [13]. Indeed, associations between metabolite concentrations and risk of diseases, e.g., cardiovascular events [14-16], diabetes [17] and some cancers [18,19], have been reported. A better understanding of how metabolite profiles differ between diet groups (defined based on the type of animal products consumed) will support mechanistic research on diet and disease risk. Previous studies, including research conducted within the Oxford arm of the European Prospective Investigation into Cancer and Nutrition (EPIC-Oxford), have found that the blood metabolite profile of individuals not habitually consuming meat significantly differs from the profile of those who consume meat [20-24]. We observed that the plasma metabolite profile, assessed using a targeted mass spectrometry (MS) assay (AbsoluteID $Q^{\circledR}$ p180 Kit, Biocrates Life Sciences AG, Innsbruck, Austria; 118 metabolites, 379 men), differed between meat-eaters, fish-eaters, vegetarians and especially vegans, primarily due to lower concentrations of glycerophospholipids and sphingomyelins in vegans [20] as well as differences in concentrations of acylcarnitines and amino acids between diet groups [20,21]. Three smaller studies ( $n=120,36$ and 31 individuals), which investigated circulating metabolite profiles of habitual diet groups, using nuclear magnetic resonance profiles (NMR; $800 \mathrm{MHz}$ using a Bruker Avance III HD spectrometer; 70 metabolites) or MS (liquid- and/or gas-chromatography coupled with MS; 66 metabolites), have reported differences between diet groups in amino acids, fatty acids, creatine, trimethylamine and xenobiotics [22-24]. Similarly, differences in the urine metabolome between individuals from different diet groups have been reported [25,26].

Here, we aimed to compare serum metabolite profiles between male meat-eaters, fish-eaters, vegetarians and vegans from EPIC-Oxford, using a high-throughput NMR platform (Nightingale Health Ltd., Helsinki, Finland), covering other parts of the blood metabolome than previous studies. To further aid planning and interpretation of studies of diet, metabolites and health outcomes, we additionally aimed to compare the measurements obtained using the NMR platform to those from MS (Biocrates AbsoluteID $Q^{\circledR} \mathrm{p} 180$ Kit) [20], clinical chemistry and capillary gas-liquid chromatography (GC) [27,28]. To our knowledge, this is the first study to characterize diet groups using the Nightingale platform and to compare metabolite levels measured using the Nightingale and Biocrates platforms. Both platforms are commonly used in prospective studies of metabolomics and disease risk [29], and the Nightingale platform measures a large number of metabolites which have not yet been studied in relation to diet groups, including lipoprotein subclass profiling with lipid concentrations within 14 subclasses.

\section{Results}

\subsection{Participant and Sample Characteristics}

This cross-sectional analysis included 286 men categorized as meat-eaters (who eat meat), fish-eaters (who eat fish but not meat), vegetarians (who eat neither meat nor fish) and vegans (who do not eat meat, fish, dairy or eggs, Figure S1). Participant characteristics and factors related to blood collection and handling by diet group are shown in Table 1. Meat-eaters had the highest body mass index (BMI) and waist circumference, and were most likely to be current smokers, while vegans were most likely to be very physically active and to drink less alcohol. Vegans had the lowest intakes of energy and saturated fatty acids (SFA), and the highest intakes of carbohydrate and polyunsaturated fatty acids (PUFA). Intake of dietary docosahexaenoic acid (DHA; C22:6 n-3, mainly from intake of fatty fish) was negligible in vegetarians and assumed to be zero in vegans. Most blood samples were taken before lunch time $(1 \mathrm{pm})$, and at blood collection meat-eaters had most recently had their last meal, followed by fish-eaters, vegetarians and vegans. Most blood samples from meat-eaters arrived at the laboratory by the end of the next working day; so did about half of the samples from vegetarians and vegans while the percentage was lowest in fish-eaters. 
Table 1. Participant characteristics, nutrient intakes and factors related to blood collection and handling by diet group ${ }^{1}$.

\begin{tabular}{|c|c|c|c|c|}
\hline & Meat-Eaters $(n=80)$ & Fish-Eaters $(n=69)$ & Vegetarians $(n=74)$ & Vegans $(n=63)$ \\
\hline \multicolumn{5}{|c|}{ Participant characteristics } \\
\hline $\begin{array}{c}\text { Age at blood collection, } \\
\text { years }\end{array}$ & $44.0(37.0,44.0)$ & $43.0(38.0,46.0)$ & $44.0(36.0,44.0)$ & $42.0(38.0,46.0)$ \\
\hline Body mass index ${ }^{2}, \mathrm{~kg} / \mathrm{m}^{2}$ & $24.5(22.1,26.1)$ & $22.7(21.1,24.4)$ & $22.9(21.6,25.7)$ & $22.1(20.5,24.4)$ \\
\hline Waist circumference ${ }^{2}, \mathrm{~cm}$ & $86.0(81.0,91.0)$ & $81.0(79.0,86.0)$ & $84.0(81.0,86.0)$ & $81.0(76.0,89.0)$ \\
\hline Current smoker & $10(12 \%)$ & $6(9 \%)$ & $5(7 \%)$ & $3(5 \%)$ \\
\hline Very physically active ${ }^{2,3}$ & $19(25 \%)$ & $12(18 \%)$ & $11(16 \%)$ & $21(35 \%)$ \\
\hline Alcohol intake, g/d & $9.8(3.2,17.5)$ & $10.6(4.9,30.0)$ & $10.6(5.1,28.6)$ & $2.9(1.0,13.0)$ \\
\hline \multicolumn{5}{|c|}{ Nutrient intakes ${ }^{4}$} \\
\hline Energy, kJ/d & $9562(8156,11508)$ & $9681(8347,11025)$ & $9640(8197,11523)$ & $8169(6615,9689)$ \\
\hline Protein, \%E & $14.0(12.7,15.7)$ & $13.0(11.6,14.6)$ & $12.6(11.1,13.9)$ & $12.6(11.2,13.8)$ \\
\hline Carbohydrate, \%E & $51.5(47.9,55.7)$ & $51.8(49.0,58.4)$ & $53.9(48.8,58.1)$ & $58.8(54.9,64.0)$ \\
\hline Total fat, $\% \mathrm{E}$ & $31.6(28.3,34.8)$ & $31.9(26.6,35.4)$ & $31.2(28.7,34.9)$ & $28.6(22.9,33.9)$ \\
\hline SFA, $\% \mathrm{E}$ & $10.2(8.7,12.1)$ & $9.7(7.7,11.8)$ & $9.8(8.0,11.8)$ & $5.4(4.2,6.8)$ \\
\hline MUFA, \%E & $11.0(9.1,11.9)$ & $10.2(8.9,11.7)$ & $10.7(9.2,11.9)$ & $10.7(7.8,12.2)$ \\
\hline PUFA, \%E & $7.2(6.2,8.1)$ & $7.9(6.8,8.8)$ & $8.0(6.8,9.1)$ & $9.6(7.9,11.4)$ \\
\hline DHA (C22:6 n-3), \%E & $0.031(0.021,0.044)$ & $0.028(0.020,0.043)$ & $0.003(0.002,0.005)$ & - \\
\hline LA (C18:2 n-6), \%E & $6.4(5.5,7.1)$ & $7.0(6.0,7.8)$ & $7.0(6.0,8.2)$ & $8.3(6.8,9.6)$ \\
\hline \multicolumn{5}{|c|}{ Blood sample related factors } \\
\hline Time since last meal ${ }^{2}, \mathrm{~h}$ & $1.8(1.0,3.3)$ & $2.0(1.2,4.0)$ & $2.3(1.5,4.0)$ & $2.5(1.5,4.1)$ \\
\hline Meds./supplements taken ${ }^{2}$ & $54(68 \%)$ & $44(65 \%)$ & $51(69 \%)$ & $40(63 \%)$ \\
\hline Time, collection ${ }^{2}$, hh:mm & $11: 10(10: 00,15: 20)$ & $10: 50(9: 45,15: 38)$ & $10: 25(9: 40,13: 00)$ & $10: 30(9: 35,15: 00)$ \\
\hline Process delay $\leq 32 \mathrm{~h}^{2,5}$ & $52(66 \%)$ & $24(35 \%)$ & $37(51 \%)$ & $27(46 \%)$ \\
\hline
\end{tabular}

${ }^{1}$ Values are median (inter-quartile range) and $\mathrm{n}(\%)$ for continuous and categorical variables, respectively. ${ }^{2}$ Information was missing for some participants; percentages or medians were calculated without these. ${ }^{3}$ Defined according to a modified version of the Cambridge Physical Activity Index [30]. ${ }^{4}$ All nutrient intakes were estimated using the National Nutrient Database for Standard Reference of the United States, as described earlier [31], and may not exactly match those previously reported for this study population [20], which were estimated using the McCance and Widdowson's The Composition of Foods. ${ }^{5}$ Samples were sent by post to the laboratory; process delay $\leq$ $32 \mathrm{~h}$ corresponds to samples which arrived by the end of the next day. Abbreviations: DHA, docosahexaenoic acid (C22:6 n-3); LA, linoleic acid (C18:2 n-6); Meds., medication; MUFA, monounsaturated fatty acids; PUFA, polyunsaturated fatty acids; SFA, saturated fatty acids; $\% \mathrm{E}$, percent of energy.

We included 207 NMR metabolite measures in the analyses (Figure S1) and correlations between pairs of these metabolites are shown in Table S1.

The Principal Component Partial R-squared (PC-PR2) analysis [32] showed that together diet group, lifestyle and blood sampling and handling related variables explained $23.1 \%$ of the total variability in the NMR data. BMI explained the highest proportion (4.3\%), after accounting for all other variables in the model. This was followed by use of medication or supplements on the day of blood collection (3.9\%) and diet group (3.4\%; Figure 1 ). Alcohol intake, physical activity, smoking status, age and time from blood collection to processing explained from 2.3 to $1.5 \%$, while lower proportions were explained by fasting status $(0.6 \%)$ and waist circumference $(0.5 \%)$. 


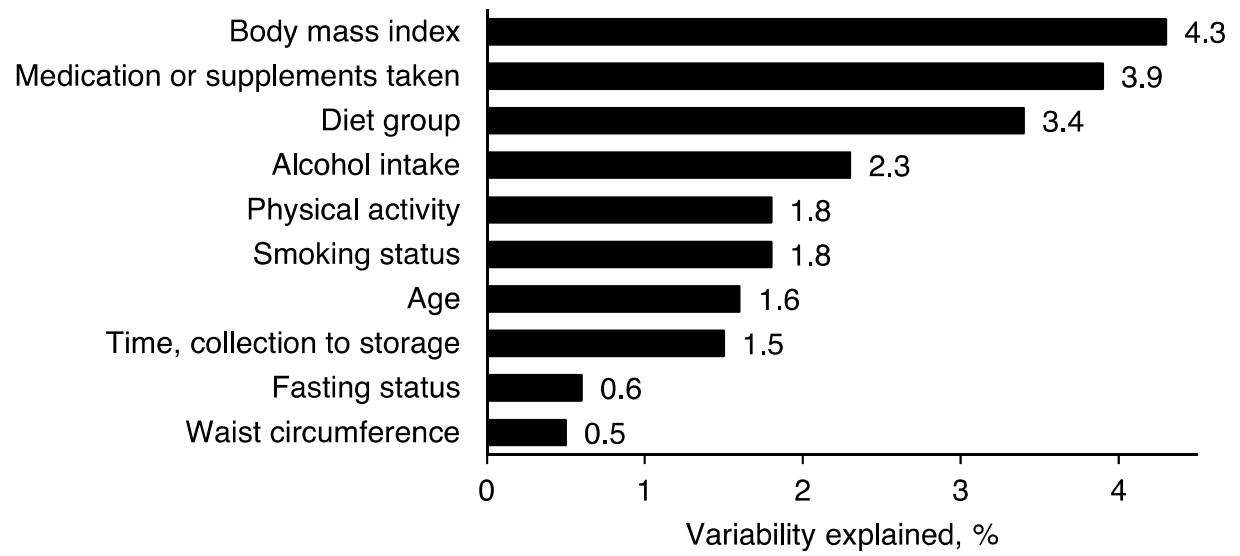

Figure 1. Variability in NMR metabolite data explained by diet group and other covariates. The percentage of the total variability explained by each variable was calculated using the Principal Component Partial R-square (PC-PR2) method [32], which accounts for all variables in the model.

\subsection{Metabolomics Profile by Diet Group —Univariate Analysis}

Of the 207 NMR metabolite variables, 104 (50.2\%) varied statistically significantly by diet group in the analysis of variance (ANOVA) after adjustment for confounding factors and correcting for multiple testing (Tables S2 and S3 shows the geometric mean metabolite measures on the original scale and standardised to the adjusted mean in meateaters, respectively). The metabolites that differed between diet groups included all lipid-related traits in the lipoprotein subclasses chylomicrons and extremely large (XXL) very-low-density lipoprotein (VLDL), large (L) VLDL and very large (XL) HDL (Highdensity lipoprotein), and all lipid-related traits except triglycerides for the subclass very small (XS) VLDL, intermediate-density lipoprotein (IDL) and L low-density lipoprotein (LDL). For all these, the lowest concentrations were observed in vegans and the highest were found in meat-eaters, except for XXL VLDL, and L VLDL, for which vegetarians had the highest concentrations. Moreover, all measurements differed significantly by diet group in the categories mean particle diameter, ratios of fatty acids to total fatty acids, and amino acids, except isoleucine and phenylalanine. Creatinine was highest in meat-eaters and lowest in vegans, with intermediate levels in fish-eaters and vegetarians.

Results for the 23 most significant associations ( $p$-value after adjusting for multiple testing $[p(a d j)]<0.001)$ are displayed in Figure 2 and Table 2. 


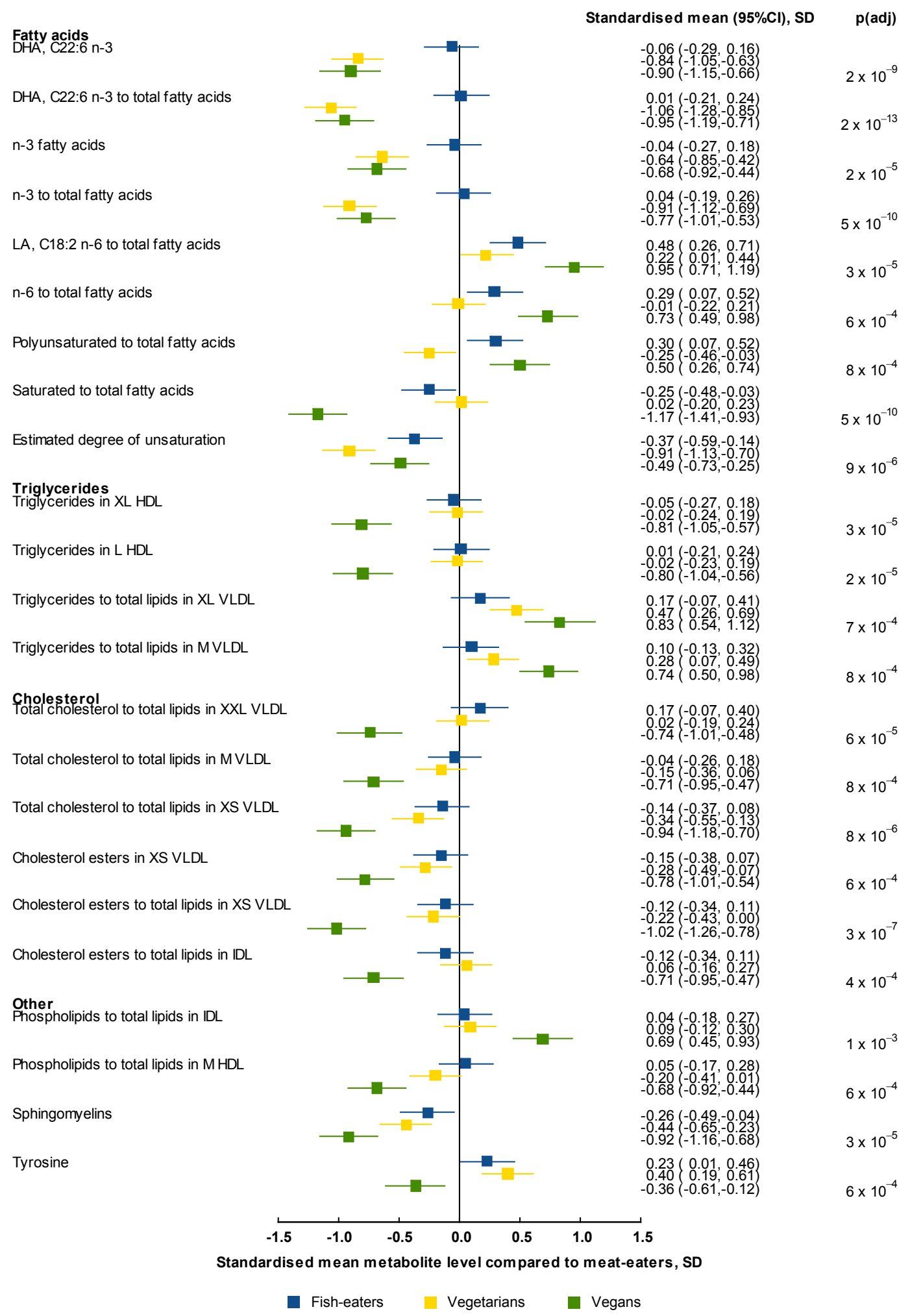

Figure 2. Geometric mean of selected NMR metabolite measures by diet group compared to meat-eaters. Means were standardized to the adjusted means in meat-eaters. $p(a d j)$ is the $p$-value after adjusting for multiple testing using a FDR of 0.05 on all 207 metabolite variables, and metabolites with $p(a d j)<0.001$ were included in the figure. All analyses were adjusted for age, BMI, smoking, alcohol, physical activity level, time since last meal at blood collection and time from blood collection to processing. Results for all NMR metabolite measures are shown in Table S3. Abbreviations: CI, confidence interval; DHA, docosahexaenoic acid; HDL, high-density lipoprotein; IDL, intermediate-density lipoprotein; L, large; LA, linoleic acid; M, medium; SD, standard deviation; VLDL, very-low-density lipoprotein; XL, extra-large; XS, extra-small; XXL, chylomicrons and extremely large. 
Table 2. Geometric mean of selected NMR metabolites by diet group on the original scale ${ }^{1}$.

\begin{tabular}{|c|c|c|c|c|c|}
\hline \multirow{2}{*}{ Metabolites } & $\begin{array}{l}\text { Meat-Eaters } \\
\quad(n=80)\end{array}$ & $\begin{array}{l}\text { Fish-Eaters } \\
\quad(n=69)\end{array}$ & $\begin{array}{l}\text { Vegetarians } \\
\quad(n=74)\end{array}$ & $\begin{array}{l}\text { Vegans } \\
(n=63)\end{array}$ & \multirow{2}{*}{$p(a d j)^{2}$} \\
\hline & Mean $(95 \%$ CI) & Mean $(95 \% \mathrm{CI})$ & Mean $(95 \%$ CI) & Mean $(95 \% \mathrm{CI})$ & \\
\hline $\begin{array}{l}\text { XS VLDL, } \mu \mathrm{mol} / \mathrm{L} \\
\text { Cholesterol esters } \\
\text { XL HDL } \mu \mathrm{mol} / \mathrm{L}\end{array}$ & $159(151,168)$ & $154(145,162)$ & $149(141,157)$ & $132(124,140)$ & $6 \times 10^{-4}$ \\
\hline $\begin{array}{l}\text { Triglycerides } \\
\text { L HDL, } \mu \mathrm{mol} / \mathrm{L}\end{array}$ & $11.4(9.95,13.1)$ & $11.1(9.62,12.8)$ & $11.2(9.85,12.8)$ & $6.89(5.93,8.00)$ & $3 \times 10^{-5}$ \\
\hline $\begin{array}{l}\text { Triglycerides } \\
\text { Ratio to total lipids in XXL } \\
\text { VLDL, } \%\end{array}$ & $20.3(17.9,23.0)$ & $20.4(18.0,23.2)$ & $20.1(17.8,22.7)$ & $12.9(11.3,14.8)$ & $2 \times 10^{-5}$ \\
\hline $\begin{array}{c}\text { Total cholesterol }{ }^{3} \\
\text { Ratio to total lipids in XL } \\
\text { VLDL, \% }\end{array}$ & $17.1(15.9,18.3)$ & $18.0(16.7,19.3)$ & $17.2(16.1,18.4)$ & $13.6(12.6,14.8)$ & $6 \times 10^{-5}$ \\
\hline $\begin{array}{c}\text { Triglycerides }{ }^{3} \\
\text { Ratio to total lipids in M } \\
\text { VLDL, \% }\end{array}$ & $60.2(59.0,61.3)$ & $61.0(59.8,62.3)$ & $62.6(61.5,63.7)$ & $64.5(63.0,66.1)$ & $7 \times 10^{-4}$ \\
\hline Total cholesterol & $28.6(27.8,29.3)$ & $28.4(27.7,29.2)$ & $28.0(27.3,28.8)$ & $26.2(25.5,27.0)$ & $8 \times 10^{-4}$ \\
\hline $\begin{array}{c}\text { Triglycerides } \\
\text { Ratio to total lipids in XS } \\
\text { VLDL, \% }\end{array}$ & $50.8(50.1,51.5)$ & $51.1(50.4,51.8)$ & $51.6(51.0,52.3)$ & $53.1(52.3,53.8)$ & $8 \times 10^{-4}$ \\
\hline Total cholesterol & $49.4(48.9,50.0)$ & $49.1(48.6,49.6)$ & $48.6(48.2,49.1)$ & $47.3(46.7,47.8)$ & $8 \times 10^{-6}$ \\
\hline Cholesterol esters & $33.6(33.1,34.1)$ & $33.3(32.9,33.8)$ & $33.1(32.7,33.6)$ & $31.5(31.0,31.9)$ & $3 \times 10^{-7}$ \\
\hline Ratio to total lipids in IDL, $\%$ & & & & & \\
\hline $\begin{array}{l}\text { Phospholipids } \\
\text { Cholesterol esters }\end{array}$ & $\begin{array}{l}27.5(27.3,27.6) \\
43.9(43.5,44.4)\end{array}$ & $\begin{array}{l}27.5(27.3,27.7) \\
43.7(43.3,44.1)\end{array}$ & $\begin{array}{l}27.5(27.4,27.7) \\
44.1(43.7,44.5)\end{array}$ & $\begin{array}{l}60.2(59.6,60.8) \\
42.6(42.2,43.1)\end{array}$ & $\begin{array}{l}1 \times 10^{-3} \\
4 \times 10^{-4}\end{array}$ \\
\hline $\begin{array}{c}\text { Ratio to total lipids in } M \\
\text { HDL, } \%\end{array}$ & & & & & \\
\hline $\begin{array}{l}\text { Phospholipids } \\
\text { Triglycerides and }\end{array}$ & $47.1(46.7,47.5)$ & $47.2(46.8,47.6)$ & $46.8(46.4,47.1)$ & $46.0(45.6,46.4)$ & $6 \times 10^{-4}$ \\
\hline $\begin{array}{l}\text { phospholipids, } \mu \mathrm{mol} / \mathrm{L} \\
\text { Sphingomyelins }{ }^{3} \\
\text { Fatty acids, } \mu \mathrm{mol} / \mathrm{L}\end{array}$ & $391(378,405)$ & $376(363,389)$ & $366(354,378)$ & $339(327,352)$ & $3 \times 10^{-5}$ \\
\hline $\begin{array}{l}\text { Estimated degree of } \\
\text { unsaturation }\end{array}$ & $1.19(1.18,1.19)$ & $1.17(1.16,1.18)$ & $1.15(1.14,1.16)$ & $1.17(1.16,1.18)$ & $9 \times 10^{-6}$ \\
\hline $\begin{array}{l}\text { DHA, C22: } 6 \text { n- } 3^{3} \\
\text { n-3 fatty acids }{ }^{3}\end{array}$ & $\begin{array}{l}126(118,134) \\
380(355,407)\end{array}$ & $\begin{array}{l}124(116,132) \\
376(350,403)\end{array}$ & $\begin{array}{c}99.3(93.4,106) \\
312(292,334)\end{array}$ & $\begin{array}{c}97.5(91.0,104) \\
307(285,331)\end{array}$ & $\begin{array}{l}2 \times 10^{-9} \\
2 \times 10^{-5}\end{array}$ \\
\hline $\begin{array}{c}\text { Ratios of fatty acids to total } \\
\text { fatty acid, } \%\end{array}$ & & & & & \\
\hline DHA, C22:6n-3 $3^{3}$ & $1.27(1.21,1.33)$ & $1.27(1.21,1.33)$ & $1.01(0.96,1.05)$ & $1.03(0.98,1.09)$ & $2 \times 10^{-13}$ \\
\hline LA, C18:2n- $6^{3}$ & $28.3(27.5,29.1)$ & $30.1(29.2,30.9)$ & $29.1(28.3,29.9)$ & $31.9(30.9,32.9)$ & $3 \times 10^{-5}$ \\
\hline$n-3$ fatty acids ${ }^{3}$ & $3.82(3.65,4.00)$ & $3.85(3.67,4.03)$ & $3.16(3.03,3.31)$ & $3.25(3.09,3.42)$ & $5 \times 10^{-10}$ \\
\hline n- 6 fatty acids ${ }^{3}$ & $33.5(33.0,34.1)$ & $34.3(33.7,34.9)$ & $33.5(33.0,34.1)$ & $35.5(34.8,36.1)$ & $6 \times 10^{-4}$ \\
\hline Polyunsaturated fatty acids ${ }^{3}$ & $37.4(36.8,38.0)$ & $38.2(37.6,38.9)$ & $36.7(36.2,37.3)$ & $38.8(38.1,39.5)$ & $8 \times 10^{-4}$ \\
\hline Saturated fatty acids ${ }^{3}$ & $36.3(36.0,36.6)$ & $36.0(35.7,36.3)$ & $36.3(36.0,36.6)$ & $34.7(34.4,35.1)$ & $5 \times 10^{-10}$ \\
\hline $\begin{array}{c}\text { Amino acids, } \mu \mathrm{mol} / \mathrm{L} \\
\text { Tyrosine }^{3}\end{array}$ & $54.8(52.5,57.1)$ & $57.2(54.8,59.7)$ & $59.1(56.7,61.6)$ & $51.1(48.8,53.5)$ & $6 \times 10^{-4}$ \\
\hline
\end{tabular}

${ }^{1}$ All analyses were adjusted for age, BMI, smoking, alcohol, physical activity level, time since last meal at blood collection and time from blood collection to processing. ${ }^{2} p(a d j)$ is the $p$-value after adjusting for multiple testing using a FDR of 0.05 on all 207 metabolite variables. Metabolite measures with $p(a d j)<0.001$ are shown. Results for all NMR metabolites are shown in Table S2. ${ }^{3}$ Missing values for some participants. Abbreviations: CI, confidence interval; DHA, docosahexaenoic acid; HDL, high-density lipoprotein; IDL, intermediate-density lipoprotein; L, large; LA, linoleic acid; M, medium; SD, standard deviation; VLDL, very-low-density lipoprotein; XL, extra-large; XS, extra-small; XXL, chylomicrons and extremely large.

Fatty acid concentrations and their ratios to total fatty acids showed some of the largest and most statistically significant differences between diet groups. For DHA and total n-3 fatty acids concentrations, and the corresponding ratios, meat-eaters and fish-eaters had the highest levels, with markedly lower levels in vegetarians and vegans. The ratios of linoleic acid (LA; C18:2 n-6) and total $n-6$ to total fatty acids were highest in vegans followed by fish-eaters, then vegetarians and meat-eaters. The ratio of SFA to total fatty acids was markedly lower in vegans than the other diet groups. The mean ratio in vegans was 1.17 ( $95 \%$ confidence interval $0.93,1.41$ ) standard deviations (SD) lower than that of meat-eaters, which was the most extreme difference observed in the study; the result for fish-eaters was $-0.25 \mathrm{SD}(-0.48,-0.03)$ and it was $0.02 \mathrm{SD}(-0.20,0.23)$ for vegetarians (Figures 2 and S3). 
Furthermore, we observed the ratio of PUFA to total fatty acids and the degree of saturation among the top hits; both were lowest in vegetarians and highest in vegans and meat-eaters, respectively.

Triglyceride concentrations in various lipoprotein subtypes were lowest in vegans, with the strongest association observed in XL and L HDL for which we observed similar higher concentrations in meat-eaters, fish-eaters and vegetarians. For the ratio between triglycerides and total lipids, the difference by diet group depended on the lipoprotein subtype; vegans had the highest for all sizes of VLDL with consecutively lower values in vegetarians, fish-eaters and meat-eaters (except for XXL VLDL), while vegans had the lowest and vegetarians the largest ratios for XL HDL and L HDL.

Total cholesterol and its ratio to total lipids were mostly highest in meat-eaters and lowest in vegans. The largest difference was observed for the ratios of total cholesterol to total lipids in XXL VLDL, medium (M) VLDL and XS VLDL, for which ratios were highest in meat-eaters and fish-eaters, similar or slightly lower in vegetarians, and markedly lower in vegans. A similar pattern was seen for cholesterol esters and their ratio to total lipids, with the most pronounced differences by diet group being in cholesterol esters in XS VLDL, the ratios in XS VLDL and in IDL.

While the ratio of phospholipids to total fatty acids in IDL was higher in vegans and similarly lower in the other diet groups, the opposite was the case for the same ratio in $\mathrm{M}$ HDL. The concentration of sphingomyelins was highest in meat-eaters, followed by fisheaters, then vegetarians and lowest in vegans. Among the amino acids, tyrosine showed the strongest variation by diet group; the concentrations were highest in vegetarians, followed by fish-eaters, then meat-eaters and lowest in vegans.

\subsection{Metabolomics Profile by Diet Group-Multivariate Analysis}

The multivariate analyses included 71 meat-eaters, 56 fish-eaters, 69 vegetarians and 41 vegans with complete NMR metabolite measurements.

From the principal component analysis (PCA), the first two principal components explained $39.4 \%$ and $22.6 \%$ of the total variation in the NMR data, respectively. The score plots showed little separation between diet groups for the principal components (Figure 3). The loadings of each individual metabolite variable on the first two principal components are shown in Table S4.

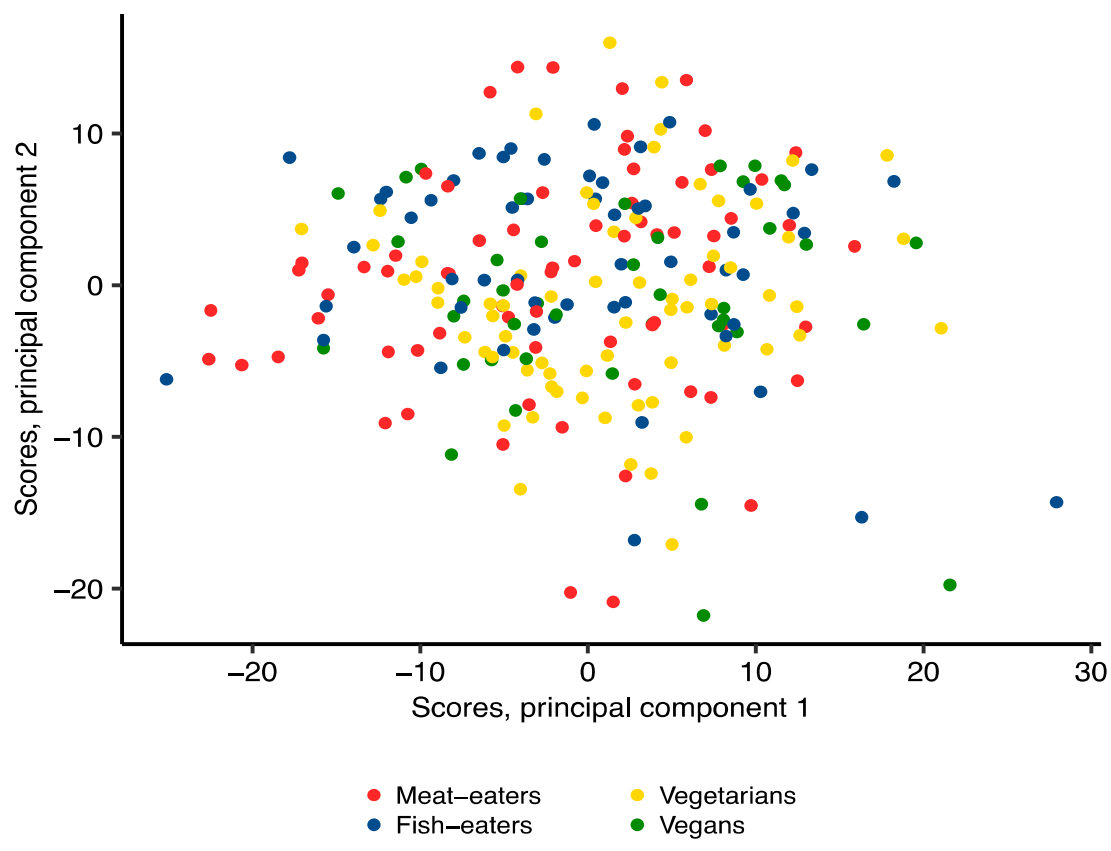

Figure 3. Score plot of principal component analysis of 207 NMR metabolite variables by diet group. 
The best partial least squares (PLS) regression model for the four diet groups contained seven components, explaining a total of $85.2 \%$ of the total variance in metabolite data, but it had low goodness of fit $\left(R^{2} Y=0.323\right)$ and goodness of prediction $\left(Q^{2} Y=0.185\right.$; Figure S2). No sign of overfitting was observed (Figure S3). The score plot of components 1 and 2 showed some separation of diet groups; meat- and fish-eaters had similar scores, while vegans separated somewhat from the other diet groups, with intermediate values in vegetarians (Figure 4a). The metabolite measures most important for differentiating the diet groups included fatty acids (absolute concentrations and/or ratios to total fatty acids of DHA [highest variable influence on projection (VIP) score of 2.2], total n-3, SFA, estimated degree of unsaturation, LA, total n-6 and PUFA), creatinine, amino acids (mainly alanine, glutamine and tyrosine), components of HDL subclasses (mainly small (S) and M HDL) and VLDL subclasses, LDL diameter, and sphingomyelins, albumin and apolipoprotein A-I concentrations (Figure $4 \mathrm{~b}$,c; Table S5).

When comparing only meat-eaters and vegans (the most extreme groups), the best model included six components and showed higher goodness of fit $\left(R^{2} Y=0.803\right)$ and prediction $\left(Q^{2} Y=0.642\right.$; Figure $\left.S 4\right)$, than when all four diet groups were included, and also no sign of overfitting (Figure S5). This model explained $83.3 \%$ of the total variance in metabolite data. The score plot of components 1 and 2 showed good separation, with vegans having higher scores on both components than meat-eaters (Figure 5a). The metabolites mostly responsible for this separation included fatty acids (mainly ratio of SFA to total fatty acids [highest VIP score of 2.45], absolute concentration and/or ratio to total fatty acids for LA, total n-6 fatty acids, PUFA and DHA), creatinine, lipid fractions in VLDL subtypes (mainly total cholesterol, cholesterol esters and free cholesterol in XS or S VLDL), amino acids (mainly alanine), various lipid-related traits in HDL subclasses (mainly of L and XL HDL), diameter of LDL and HDL particles, apolipoprotein A-I, phospholipids (i.e., sphingomyelins, phosphatidylcholines and other cholines, total cholines, and total phosphoglycerides), and the inflammatory marker glycoprotein acetyls (Figure 5b,c; Table S5). 


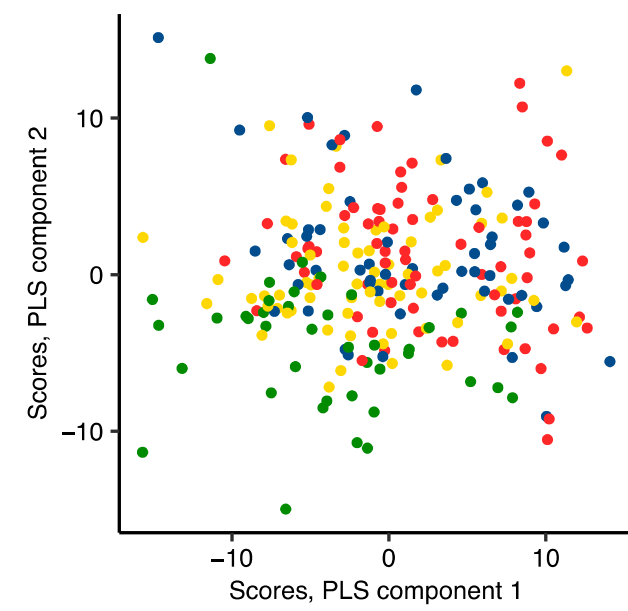

Meat-eaters Vegetarians

- Fish-eaters Vegans

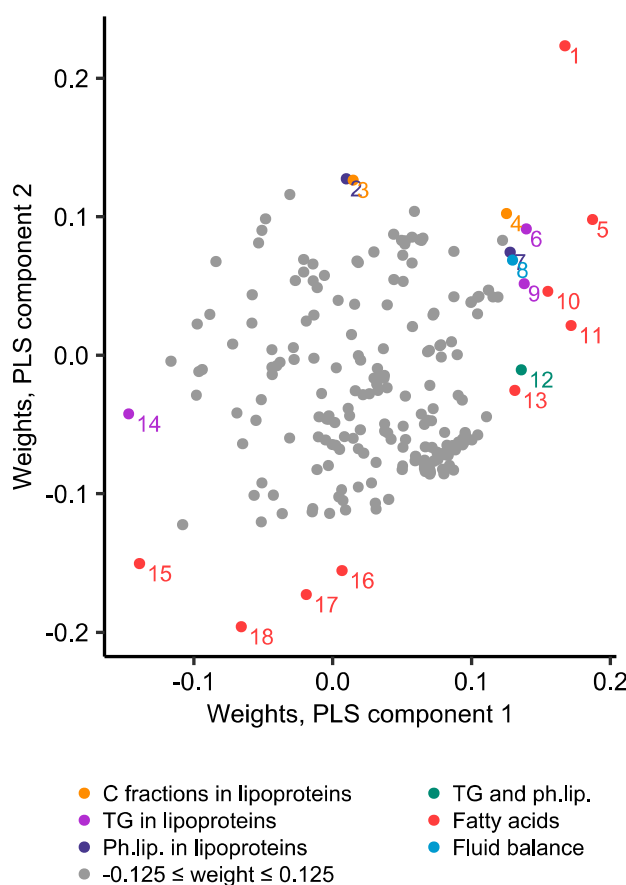

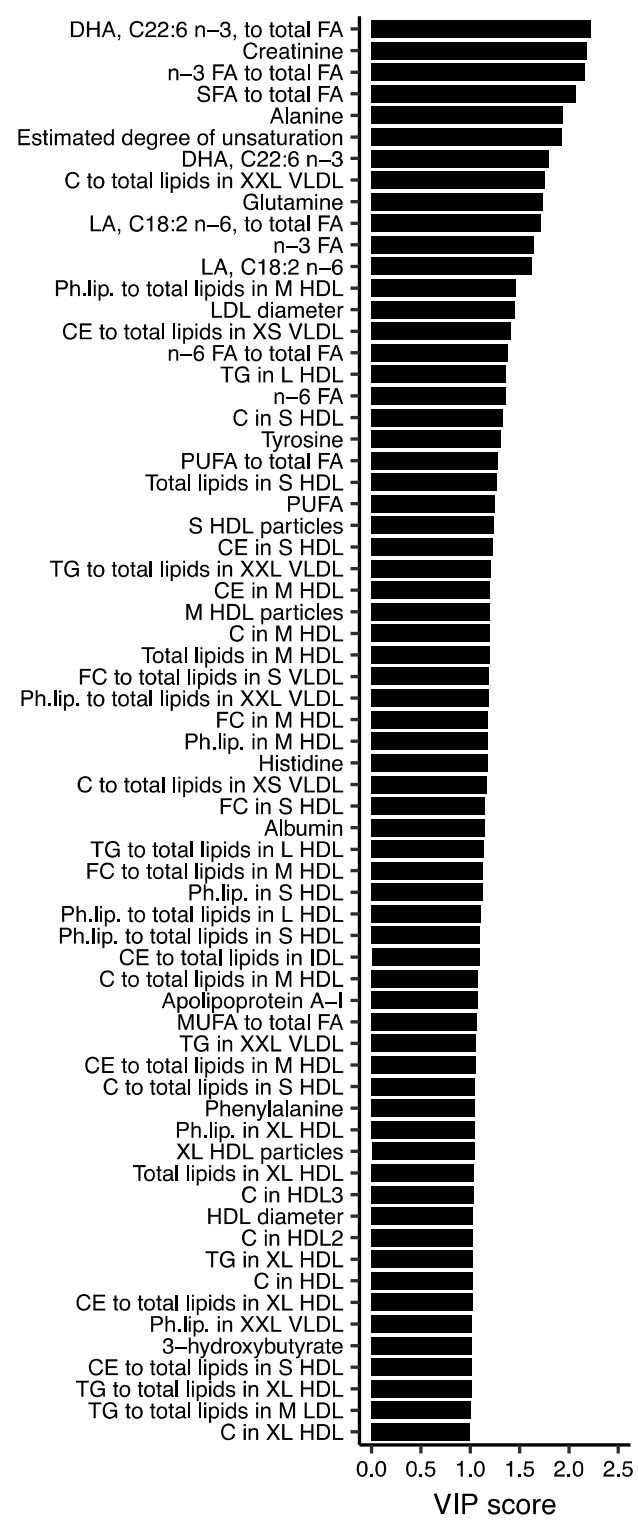

Figure 4. PLS model of 207 NMR metabolite measures by four diet groups. (a) Score plot of PLS components 1 and 2 coloured by diet group; (b) Weights plot of PLS components 1 and 2. Metabolites with weights more extreme than \pm 0.125 on either PLS component are coloured and labelled: 1, Saturated to total fatty acids; 2, Phospholipids to total lipids in S HDL; 3, Free cholesterol to total lipids in S HDL; 4, Cholesterol esters to total lipids in XS VLDL; 5, DHA, C22:6 n-3, to total fatty acids; 6, Triglycerides in L HDL; 7, Phospholipids to total lipids in M HDL; 8, Creatinine; 9, Triglycerides in XL HDL; 10, n-3 to total fatty acids; 11, DHA, C22:6 n-3; 12, Sphingomyelins; 13, n-3 fatty acids; 14, Triglycerides to total lipids in XL VLDL; 15, Linoleic acid, C18:2 n-6, to total fatty acids; 16, Polyunsaturated fatty acids; 17 , n-6 fatty acids; 18 , Linoleic acid, C18:2 n-6; (c) Variable influence on projection (VIP) scores, representing the contribution of metabolites to the PLS model; metabolites with VIP $>1$ are included in the figure. Further details are given in Table S5. Abbreviations: C, total cholesterol; $\mathrm{CE}$, cholesterol esters; $\mathrm{C}$ fractions, cholesterol fractions, i.e., total cholesterol, cholesterol esters and free cholesterols; DHA, docosahexaenoic acid; FA, fatty acids; FC, free cholesterol; HDL, high-density lipoprotein; IDL, intermediate-density lipoprotein; L, large; LA, linoleic acid; LDL, low-density lipoprotein; M, medium; MUFA, monounsaturated fatty acids; Ph.lip., phospholipids; PLS, partial least squares; PUFA, poly unsaturated fatty acids; S, small; SFA, saturated fatty acids; TG, triglycerides; VIP, variable influence on projection; VLDL, very-low-density lipoprotein; XL, extra-large; XS, extra-small; XXL, chylomicrons and extremely large. 

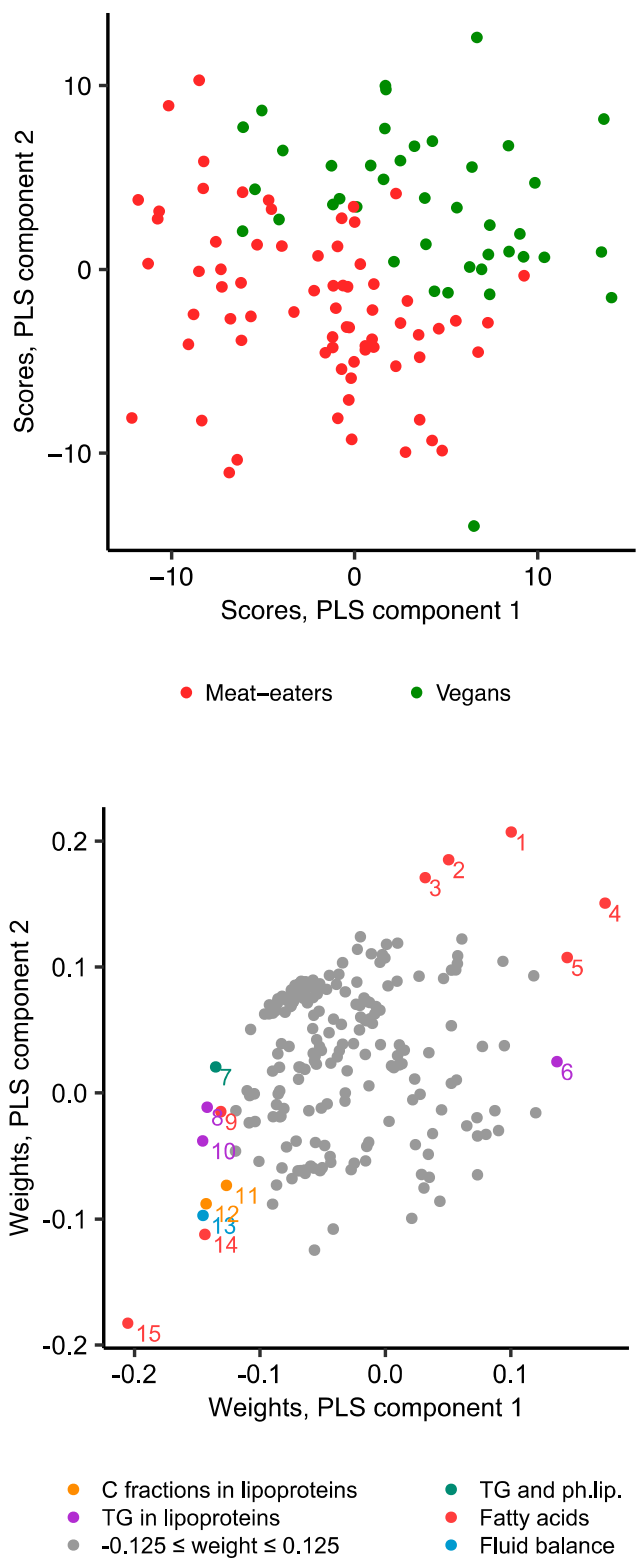

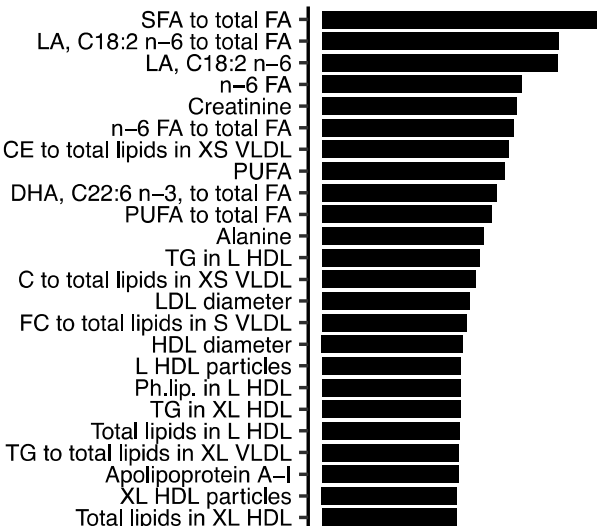

Total lipids in XL HDL al lipids in XL HDL Ph.lip. in XL HDL Sphingomyelins FC in $\mathrm{XL} H D L$ $C$ in $L$ HDL

Ph.lip. in $M \mathrm{HDL}$ $C$ in XL HDL FC to total lipids in $M$ HDL Ph.lip. to total lipids in M HDL
Ph.lip. to total lipids in S HDL $C$ in $\mathrm{HDL}$
$\mathrm{CE}$ in $\mathrm{XL} H \mathrm{HDL}$
Ph.lip. to total lipids in $\mathrm{XL} H D L$ $C$ to total lipids in XL HDL $C$ in $S \mathrm{HDL}$ FC in $L$ HDL

Glycoprotein acetyls
Total FA PC and other cholines $C$ to total lipids in XXL VLDL Total phosphoglycerides Ph.lip. to total lipids in S VLDL $F C$ in $M H D L$ , $\mathrm{C}$ in $\mathrm{HDL} 3$ Tyrosine CE to total lipids in IDL $F C$ to total lipids in $S$ HDL
$F C$ to total lipids in XL VLDL $F C$ to total lipids in XL VLDL $T G$ to total lipids in XL HDL $C$ to total lipids in $\mathrm{S} H \mathrm{HL}$ $M$ HDL particles Total lipids in M HDL CE to total lipids in S HDL
CE to total lipids in M LDL Ph.lip. in S VLDL $T G$ to total lipids in XS VLDL

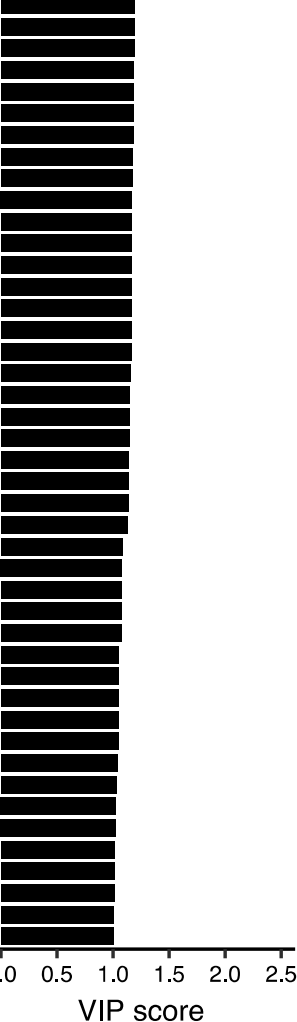

Figure 5. PLS model of 207 NMR metabolite measurements in meat-eaters and vegans. (a) Score plot of PLS components 1 and 2 coloured by diet group; (b) Weights plot of PLS components 1 and 2. Metabolites with weights more extreme than \pm 0.125 on either PLS component are coloured and labelled: 1 , Linoleic acid, C18:2 n-6; 2, n-6 fatty acids; 3, Polyunsaturated fatty acids; 4, Linoleic acid, C18:2 n-6, to total fatty acids; 5, n-6 to total fatty acids; 6 , Triglycerides to total lipids in XL VLDL; 7, Sphingomyelins; 8, Triglycerides in XL HDL; 9, DHA, C22:6 n-3; 10, Triglycerides in L HDL; 11, Total cholesterol to total lipids in XS VLDL; 12, Cholesterol esters to total lipids in XS VLDL; 13, Creatinine; 14, DHA, C22:6 n-3, to total fatty acids; 15, Saturated to total fatty acids; (c) Variable influence on projection (VIP) scores, representing the contribution of metabolites to the PLS model; metabolites with VIP $>1$ are included in the plot. Further details are given in Table S5. Abbreviations: $\mathrm{C}$, total cholesterol; $\mathrm{CE}$, cholesterol esters; $\mathrm{C}$ fractions, cholesterol fractions, i.e., total cholesterol, cholesterol esters and free cholesterols; DHA, docosahexaenoic acid; FA, fatty acids; FC, free cholesterol; HDL, high-density lipoprotein; IDL, intermediate-density lipoprotein; L, large; LA, linoleic acid; LDL, low-density lipoprotein; M, medium; MUFA, monounsaturated fatty acids; Ph.lip., phospholipids; PLS, partial least squares; PUFA, poly unsaturated fatty acids; S, small; SFA, saturated fatty acids; TG, triglycerides; VIP, variable influence on projection; VLDL, very-low-density lipoprotein; XL, extra-large; XS, extra-small; XXL, chylomicrons and extremely large. 


\subsection{Comparison of NMR Measures with Those from MS, Clinical Chemistry and GC}

The NMR metabolites overlapped with nine analytes obtained using MS (eight amino acids and creatinine), four obtained from clinical chemistry (apolipoproteins and cholesterols) and two analytes obtained from GC (fatty acids).

Between assay-method correlation coefficients ( $r$ ) for the amino acids and creatinine varied from 0.49 for phenylalanine to 0.94 for alanine (Table 3). For histidine, excluding one extreme outlier reduced the correlation from 0.74 to 0.68 . For the apolipoproteins and cholesterols correlations were high $(r \geq 0.82)$, while those for DHA and LA were low $(r \leq 0.15)$.

Table 3. Pearson correlations between NMR metabolite concentrations and those measured using another method.

\begin{tabular}{|c|c|c|c|}
\hline Metabolites & $n$ & $r$ & $p$ \\
\hline \multicolumn{4}{|l|}{ Mass spectrometry ${ }^{1}$} \\
\hline Alanine & 286 & 0.94 & $<0.0001$ \\
\hline Glutamine & 279 & 0.62 & $<0.0001$ \\
\hline Histidine & 284 & 0.74 & $<0.0001$ \\
\hline Histidine, excl. 1 outlier & 283 & 0.68 & $<0.0001$ \\
\hline Isoleucine & 286 & 0.83 & $<0.0001$ \\
\hline Leucine & 286 & 0.87 & $<0.0001$ \\
\hline Valine & 286 & 0.70 & $<0.0001$ \\
\hline Phenylalanine & 220 & 0.49 & $<0.0001$ \\
\hline Tyrosine & 284 & 0.87 & $<0.0001$ \\
\hline Creatinine & 284 & 0.84 & $<0.0002$ \\
\hline \multicolumn{4}{|l|}{ Clinical chemistry ${ }^{2}$} \\
\hline Apolipoprotein A-I & 286 & 0.82 & $<0.0001$ \\
\hline Apolipoprotein B & 286 & 0.92 & $<0.0001$ \\
\hline Total cholesterol & 286 & 0.94 & $<0.0001$ \\
\hline $\begin{array}{l}\text { Total high-density lipoprotein } \\
\text { (HDL) cholesterol }\end{array}$ & 286 & 0.86 & $<0.0001$ \\
\hline \multicolumn{4}{|l|}{$\begin{array}{l}\text { Capillary gas-liquid } \\
\text { chromatography }^{3}\end{array}$} \\
\hline $\begin{array}{c}\text { C22:6 n-3, docosahexaenoic } \\
\text { acid (DHA) }\end{array}$ & 71 & 0.03 & 0.8 \\
\hline $\begin{array}{l}\text { C22: } 6 \text { n-3, docosahexaenoic } \\
\text { acid (DHA), excl. } 1 \text { outlier }\end{array}$ & 70 & -0.01 & 0.9 \\
\hline C18:2 n-6, linoleic acid (LA) & 72 & 0.15 & 0.2 \\
\hline
\end{tabular}

${ }^{1}$ Plasma amino acid and creatinine concentrations were measured using the mass spectrometry based AbsoluteIDQ ${ }^{\circledR}$ p180 Kit (Biocrates Life Sciences AG, Innsbruck, Austria) [20]. ${ }^{2}$ In serum, apolipoproteins were measured using an immunoturbimetric assay, total cholesterol was measured using an enzymatic assay and HDL cholesterol was measured directly [27]. ${ }^{3}$ Total plasma esterified and nonesterified fatty acids were measured using capillary gas-liquid chromatography [28].

Inspection of the Bland-Altman plots showed good agreement between the NMR measurements and other measurements at low average concentrations (Figure S6 and Table S6). However, at higher average concentrations, those measured using NMR were lower than those measured using other methods.

Correlations of all metabolites measured using NMR with those measured using other methods are shown in Table S7. Correlations ranged from -0.88 (ratio of phospholipids to total lipids in L LDL with apolipoprotein B) to 0.94 (Phospholipids in M LDL with apolipoprotein B). Strong positive correlations were observed especially for NMR lipoprotein subclasses, their lipid concentrations and components with cholesterols measured using clinical chemistry, and for XS VLDL, IDL and LDL subclasses from NMR with some sphingomyelins from MS (Figure 6). Strong inverse correlations were found especially for ratios of free cholesterol, triglycerides and phospholipids to total lipids in lipoprotein subclasses from NMR with total cholesterol, apolipoprotein $B$ and non-high-density lipoprotein (non-HDL) cholesterol from clinical chemistry. The same was the case for the ratios of LA, total $\mathrm{n}-6$ fatty acids and PUFA to total fatty acids with some diacyl-phosphatidylcholines (PC aa; Figure 7). 


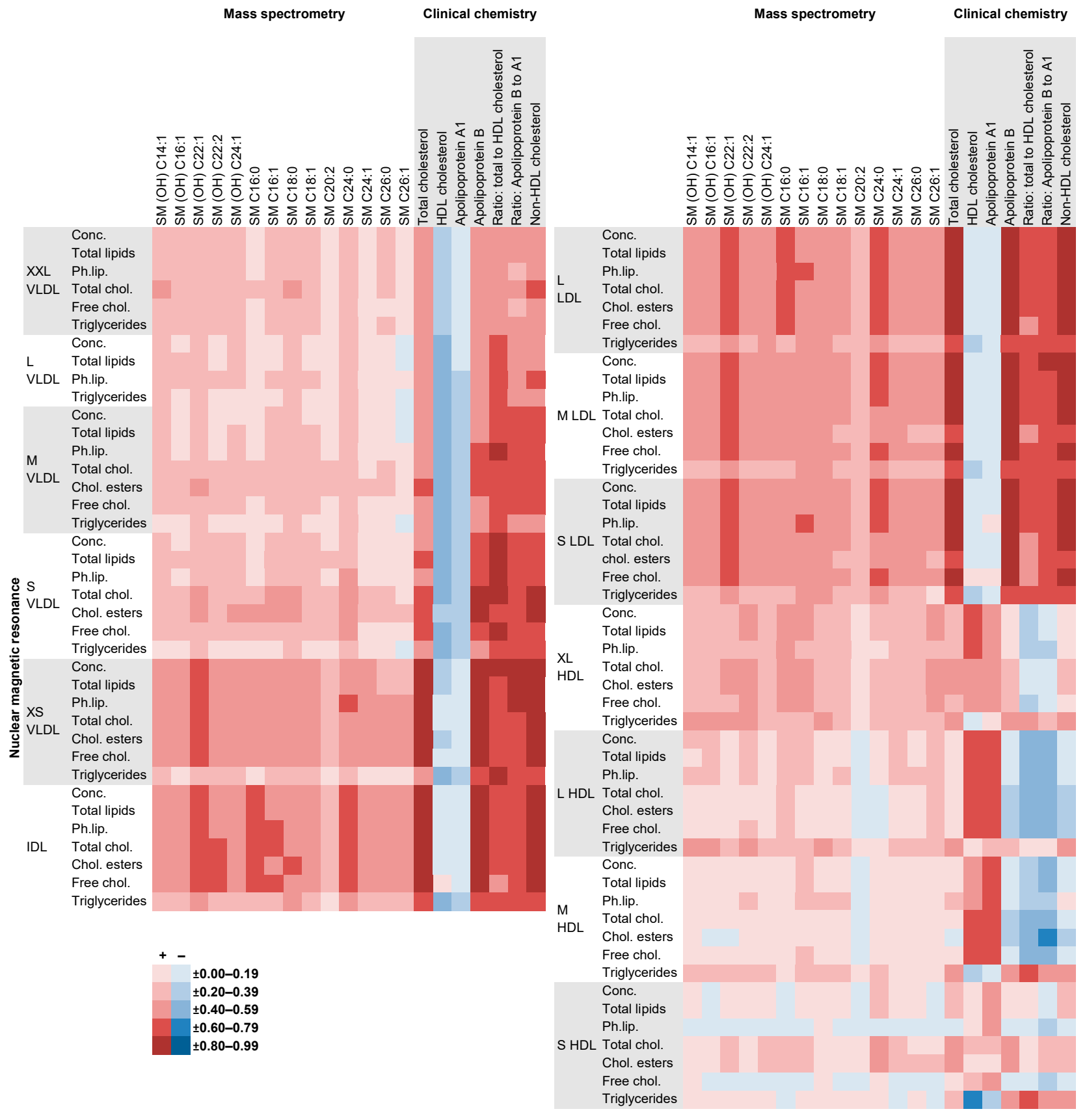

Figure 6. Correlations of NMR lipoproteins with clinical chemistry and MS sphingomyelins. Plasma sphingomyelin concentrations were measured using the mass spectrometry based AbsoluteIDQ ${ }^{\circledR}$ p180 Kit (Biocrates Life Sciences AG, Innsbruck, Austria) [20]. 'Cx:y' denotes the number of carbon atoms and double bonds in the fatty acid side chain of the sphingomyelins (SM) and hydroxy-sphingomyelins [SM $(\mathrm{OH})]$. In serum, apolipoproteins were measured using an immunoturbimetric assay, total cholesterol was measured using an enzymatic assay and HDL cholesterol was measured directly [27]. Further details are seen in Table S7. Abbreviations: Chol., Cholesterol; Conc., concentration; HDL, high-density lipoprotein; IDL, intermediate-density lipoprotein; L, large; LDL, low-density lipoprotein; M, medium; NMR, nuclear magnetic resonance; Ph.lip., phospholipids; S, small; SM, sphingomyelin; SM (OH), hydroxyl-sphingomyelin; VLDL, very-low-density lipoprotein; XL, extra-large; XS, extra-small; XXL, chylomicrons and extremely large. 


\section{NMR: Ratios of fatty acids} to total fatty acid

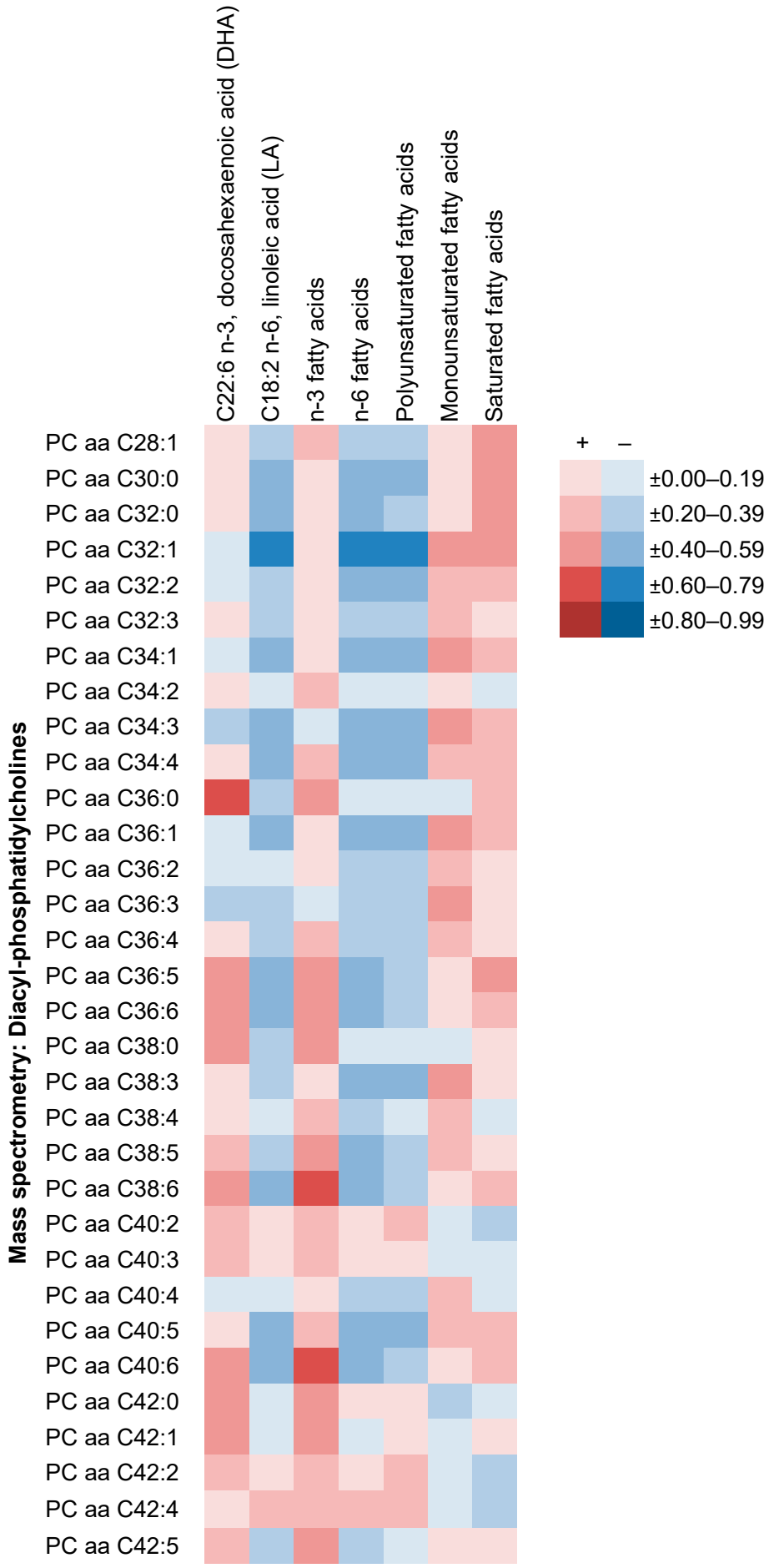

Figure 7. Correlations of NMR fatty acid ratios with MS diacyl-phosphatidylcholine concentrations. Plasma diacyl-phosphatidylcholine (PC aa) concentrations were measured using the mass spectrometry based AbsoluteIDQ ${ }^{\circledR}$ p180 Kit (Biocrates Life Sciences AG, Innsbruck, Austria) [20]. “Cx:y" denotes the total number of carbon atoms and double bonds in the two fatty acid side chains of the phosphatidylcholines. Further details are seen in Table S7. 


\section{Discussion}

\subsection{Main Findings}

In this study we compared NMR metabolite measures between four distinct diet groups and compared the NMR measures to those from a MS-based metabolomics platform, clinical chemistry and GC.

In the analysis of differences in metabolite profiles between diet groups, we consistently found differences for fatty acids. Vegans had higher levels (i.e., concentrations and / or ratios to total fatty acids) of LA, total n- 6 fatty acids and PUFA, and lower levels of DHA, total n-3 fatty acids and SFA, compared to meat-eaters. This is in accordance with previous results from this cohort [28], with other data [33] and with the observed intakes of these fatty acids (Table 1) [33]. For total n-3 fatty acids and DHA measures, vegetarians had lower levels similar to those of vegans and fish-eaters had higher levels similar to those of meat-eaters. For the other fatty acids, vegetarians and fish-eaters mostly had intermediate levels. Moreover, consistently lower concentrations of sphingomyelins, tyrosine and creatinine, and particle diameter of LDL and HDL were observed in vegans, as were higher concentrations of alanine; for all but the amino acids, meat-eaters had the highest levels, followed by fish-eaters and vegetarians. Finally, many differences were observed for various lipid fractions in VLDL, including triglycerides and cholesterols (for which concentrations were lowest in vegans), and in HDL (for which concentrations were mostly lowest in vegans). The results for HDL are in line with those previously reported in this cohort for total HDL cholesterol [27].

While the agreement between measurements of the overlapping metabolites on the NMR platform with the MS- and clinical chemistry-based methods was high for alanine, isoleucine, leucine, tyrosine, creatinine, apolipoproteins, total and HDL cholesterols $(r \geq 0.83$; in line with other data $[15,34])$, it was lower for the remaining amino acids $(r \geq 0.49)$. The very low correlations for DHA and LA concentrations $(r \leq 0.15)$ observed here are in contrast with other studies comparing the NMR protocol with gas chromatography $(r \geq 0.95)[14,35]$. However, the associations of DHA and LA measured using NMR with diet group in the current analysis were comparable with those previously observed in EPIC-Oxford [28] and elsewhere [33] (both GC), although less strong. More widely, strong correlations were observed between the metabolites covered by the NMR platform and other assay methods. This was especially the case for NMR lipoprotein subclasses and their lipid concentrations and compositions with lipids measured using clinical chemistry (e.g., apolipoprotein B), suggesting that standard clinical chemistry may capture a substantial proportion of the information provided by the NMR platform for the lipoproteins. Strong correlations were also observed for NMR lipoprotein subclasses with MS-based sphingomyelins, and for NMR metabolite measures involving PUFA with diacyl-phosphatidylcholines from MS.

\subsection{Findings in Context of the Literarure}

While some studies have compared metabolomics platforms commonly used in prospective studies using split samples [29,36-38], to our knowledge none has included both the Biocrates (MS) and Nightingale (NMR) platforms (both commonly used in prospective studies of metabolomics and disease risk [29]), as we do here. As shown, there was limited overlap between the metabolites measured by these two platforms. However, for creatinine and the eight amino acids which did overlap, we observed good to excellent between-method correlations and consistent findings by diet group between the current and our previous studies [20,21].

Differences in coverage of the metabolome by different metabolomics platforms make comparisons of results across metabolomics studies complex. Moreover, for studies of metabolite profiles by diet group, study populations differ and dietary habits within the groups are likely also to differ. However, here we compare our current findings to previous published results. One previous study has compared the blood NMR metabolite profile of diet groups, including omnivores, fish-eaters, vegetarians and vegans, as in the current 
study $\left(n_{\text {total }}=120\right)$ [23]. In contrast to our study, Lindqvist et al. included both men and women $(62.5 \%)$, used more standardised blood collection and handling procedures (including fasting samples and controlled temperature), and used another NMR technology, which measured fewer metabolites ( $\mathrm{n} 70$ ). The main findings included lower isoleucine, leucine, valine and creatine, and higher glutamine, glycine and trimethylamine in vegans compared to non-vegans. Moreover, creatinine was higher in meat-eaters than in nonmeat-eaters; total cholesterol, LDL, HDL and creatinine measured using clinical chemistry were also highest in the meat-eaters [23]. The findings for leucine, valine, glutamine, creatinine and cholesterols were in line with the current study and with our study of metabolites measured using the MS-based Biocrates platform [20]. Similarly, a small study $(n=36)$, by Wang et al., comparing 66 MS metabolites in fasted serum between three diet groups, has also reported lower concentrations of leucine and valine, as well as of isoleucine, in vegans and vegetarians compared to omnivores [24]. Glycine was not measured by the Nightingale platform or by Wang et al. [24] but Lindqvist et al.'s results [23] correspond to our previous findings [20,21]. Trimethylamine and creatine were not measured by the platforms from Nightingale or Biocrates, nor by Wang et al. [24]. Finally, while Lindqvist et al. reported that lipids (reported as one trait) were not a major contributor to the separation between diet groups, in models including men [23], various lipids (including cholesterols, fatty acids, phospholipids and triglycerides) were important for group separation in the current study, in the study by Wang et al. [24], and in our previous MS-based study [20]. Specifically, a key finding of our previous study was lower concentrations of numerous phosphatidylcholines and sphingomyelins in vegans, which were the metabolites mostly responsible for separation between diet groups [20]. In the NMR assay, these two metabolite classes were measured as part of the phospholipid components of lipoprotein subclasses and as part of total phosphoglycerides, total cholines, phosphatidylcholine and other cholines, and sphingomyelins. While concentrations of these metabolites were consistently lower in vegans, they did not have a major influence on the discrimination between diet groups in the current study.

Although we did not aim to investigate the possible mediating role of metabolites in associations between diet groups and disease outcomes, our results on NMR metabolites may help to interpret studies of the associations of diet groups with disease risk. For example, prospective studies have reported lower risk of type 2 diabetes in vegans $[9,10]$ and in individuals with higher circulating LA [39,40]. We found that vegans had higher LA levels than the other diet groups, and thus circulating LA could play a role in the association between diet group and risk of type 2 diabetes, albeit with BMI as a major contributing factor. Furthermore, several ratios of fatty acids to total fatty acids, including DHA, total n-3, LA, total n-6, SFA and PUFA, which were among the metabolite measures most strongly associated with diet group in the current study, have been linked to lower risk of cardiovascular events [14-16]. In the current study, some of these fatty acids ratios were lowest in vegetarians and vegans (DHA and total $n-3$ ), possibly suggesting a higher risk of cardiovascular disease in these diet groups, while other were highest in vegans followed by fish-eaters (LA, total n-6 and PUFA), suggesting a lower risk in these groups. This might possibly be in line with the lower risk of ischemic heart disease observed in fisheaters, and especially in vegetarians and vegans, compared to meat-eaters [4]. However, to understand the potential mediating role of these fatty acids ratios in the associations between diet group and subtypes of cardiovascular disease, further studies are needed.

\subsection{Strengths and Limitations}

The main strengths of this study are the inclusion of four distinct diet groups from a well characterized cohort, which allowed a detailed study of metabolite profile of various diets, while limiting the potential role of confounding. Moreover, we investigated two aspects important for planning and interpreting studies of metabolites in relation to disease risk and diet-disease associations. We were able to describe the largest study of differences in NMR metabolites, the first for the Nightingale platform, and to provide 
the first comparison of metabolites measured using the Nightingale and the Biocrates (MS) platforms.

There are however limitations to the study. Samples were non-fasted and were not cooled during transport to the laboratory, which may have affected the results of the NMR metabolomics assay [41,42], albeit studies exploring the potential impact of pre-processing conditions on the specific metabolites measured using the Nightingale platform are scarce. One study has reported that most of the metabolites measured using the Nightingale platform in non-fasted serum samples were not materially affected by delaying processing for $48 \mathrm{~h}$ at $21^{\circ} \mathrm{C}$ (experimental condition; conditions similar to those in the current study) compared to processing within $1.5 \mathrm{~h}$ at $4{ }^{\circ} \mathrm{C}$ (reference condition) [42]. For metabolites consistently showing up as different between diet groups in the current analysis, rank correlations between metabolite concentrations in samples kept at the experimental versus the reference condition were 0.7 or above, except for, tyrosine $(r=0.6)$ and lipid traits in small HDL (ranging from 0.6 and 0.7). Moreover, in the current analysis, fasting status and time from blood collection to processing explained only a small proportion $(0.6 \%$ and $1.5 \%$, respectively) of the total variability of NMR metabolites. Fasting status did not differ greatly between diet groups, but some differences were observed for process delay. In combination, this suggests that bias arising from pre-processing did not have much impact on our results.

A further limitation was the lack of data on the type of medication and supplements taken on the day of blood collection. We found that any use explained 3.9\% of the total variability of the NMR metabolite measures and that the proportion of participants taking any medication or supplements did not materially differ between diet groups. It has been shown that starting statin treatment is associated with changes in the measured lipoproteins concentrations and components, apolipoproteins, fatty acid concentrations and ratios, while metabolites in other classes were mainly unrelated to statin use [43]. Finally, the NMR and MS assays were done on serum and plasma samples, respectively, which may have impacted the comparability of metabolite levels measured using the two platforms [44].

\subsection{Future Work}

While this study provides insights into the comparability and complementarity of two metabolomics platforms and other assay methods, comparisons using split samples with other platforms frequently used for metabolomics studies in epidemiology are also warranted [29]. Moreover, further research on the effect of factors related to blood sample collection, handling and storage on the metabolites measured using the Nightingale NMR platform would aid interpretation of large-scale cohort data on these metabolites and disease risk. Finally, many epidemiological studies rely on a single metabolite measurement to represent long-term exposure in analyses of disease risk. To our knowledge, one study has reported poor to excellent reproducibility of the metabolites measured using the Nightingale platform in fasting serum samples over 3.6 years (median intra-class correlation coefficient of 0.66 , range 0.17 to 0.84 ) [45]; replication of these results and reproducibility over longer time periods would also provide valuable information for studies of these metabolites and disease outcomes.

\section{Materials and Methods}

\subsection{Study Population and Data Collection}

EPIC-Oxford consists of 65,000 men and women aged at least 20 years who were recruited across the United Kingdom between 1993 and 2000 [46]. The main aim of this cohort is to investigate associations between diet, lifestyle and cancer risk in individuals with different long-term dietary habits; thus, a large number of non-meat-eaters were recruited.

At recruitment, participants provided extensive information on diet, lifestyle, body size and previous disease, and 20,000 also provided a blood sample. The diet groups were defined as meat-eaters (who eat meat), fish-eaters (who eat fish but not meat), veg- 
etarians (who eat neither meat nor fish) and vegans (who do not eat meat, fish, dairy or eggs) based on "yes" /"no"-questions on intakes of meat, fish, dairy products or eggs. Moreover, participants filled in a 130-item validated semi-quantitative food frequency questionnaire $[47,48]$. Blood sample were taken at the participant's local general practitioner's surgery; participants were not required to fast. Whole blood was transported by post at ambient temperature to the laboratory where they were processed for long-term storage in liquid nitrogen $\left(-196^{\circ} \mathrm{C}\right)$ until 2011 and subsequently in electric freezers $\left(-80^{\circ} \mathrm{C}\right)$.

Participants in the current study were a subset of participants in our previous study of MS-based metabolites by diet group [20]. In brief, all participants were men between 30 and 49 years of age, with known smoking status and diet group, with reliable energy intake, and without prior major disease.

All participants gave written informed consent prior to entering the EPIC-Oxford study. The study was conducted in accordance with the Declaration of Helsinki, and the protocol for EPIC-Oxford was approved by a Multicentre Research Ethics Committee (MREC /02/0/90): Scotland A Research Ethics Committee (IRAS 223031; updated approval on $28 / 03 / 2018$ ).

\subsection{Laboratory Analysis}

A total of 225 metabolite measures, including 146 fully quantified metabolite concentrations and 79 derived variables, were obtained from serum samples using a targeted high-throughput NMR spectroscopy platform (Nightingale Health Ltd., Helsinki, Finland) [49-51]. This method provides simultaneous quantification of common lipids, lipoprotein subclass profiling with lipid concentrations within 14 subclasses, fatty acid composition, and various low-molecular weight metabolites including amino acids, ketone bodies and markers of glycolysis, fluid balance and inflammation (an overview of the spectral region of the metabolites is available in [49]). A detailed description of the sample preparation and acquisition parameters has been published previously $[49,50]$.

In brief, the frozen samples are thawed overnight in a fridge at $+4{ }^{\circ} \mathrm{C}$, mixed, and centrifuged at $3400 \times g$ for $10 \mathrm{~min}$. Thereafter, $70 \mu \mathrm{L}$ serum was mixed with $70 \mu \mathrm{L}$ sodium phosphate buffer $\left(75 \mathrm{mM} \mathrm{Na}_{2} \mathrm{HPO}_{4}\right.$ in $80 \% / 20 \% \mathrm{H}_{2} \mathrm{O} / \mathrm{D}_{2} \mathrm{O}$, pH 7.4 ; including also $0.08 \%$ sodium 3-(trimethylsilyl)propionate-2,2,3,3-d 4 and $0.04 \%$ sodium azide) for a total sample volume of $140 \mu \mathrm{L}$ in $3 \mathrm{~mm}$ outer diameter SampleJet NMR tubes. This sample preparation was done using an automated procedure (PerkinElmer JANUS Automated Workstation, Waltham, MA, USA). No extraction procedure was applied. The samples were then preheated to $37.5^{\circ} \mathrm{C}$ just prior to spectra acquisition, which was performed using a Bruker AVANCE III spectrometer operating at $500.36 \mathrm{MHz}(1 \mathrm{H}$ observation frequency, $11.74 \mathrm{~T}$; Bruker, MA, USA). Data were acquired using two NMR pulse sequences. Lipids and macromolecular data were acquired using a standard Bruker NOESY PRESAT (noesygppr1d) sequence. This was acquired with $80 \mathrm{k}$ data points using 8 transients after 4 dummy scans. The $90^{\circ}$ pulse length was automatically calibrated for each sample, the mixing time was $10 \mathrm{~ms}$, and an irradiation field of $25 \mathrm{~Hz}$ was used to suppress the water peak. The acquisition time was $2.7 \mathrm{~s}$ and the relaxation delay $3.0 \mathrm{~s}$. Low molecular weight metabolite data were acquired using a standard Bruker CPMG (cpmgpr1d) sequence. This was acquired using $64 \mathrm{k}$ data points using 16 transients after 4 dummy scans. The same automatically calibrated pulse length as in the NOESY was used for each sample, the CPMG T2-filter was $78 \mathrm{~ms}$ with a $403 \mu$ s fixed-echo delay, and an irradiation field of $25 \mathrm{~Hz}$ was used to suppress the water peak. The acquisition time was $3.3 \mathrm{~s}$ and the relaxation delay $3.0 \mathrm{~s}$. All free induction decays were phase corrected and processed in an automated fashion. The FIDs were zero-filled to $128 \mathrm{k}$ data points and then an exponential window function with $1.0 \mathrm{~Hz}$ line broadening was applied.

We included $9 \%(n=35)$ quality control samples of pooled plasma among the study samples. Laboratory personnel were blinded to diet group and quality control sample status. Metabolites with coefficients of variation from the blinded quality control samples greater than 20\% were excluded ( $n=15$; Table S8 and Figure S1). Furthermore, we excluded 
metabolites for which more than $20 \%$ of men had non-quantifiable measurements (missing or below the limit of quantification [LOQ]); $n=1$ ). Metabolite values below the LOQ were set to half the lowest measured value for each metabolite. Finally, we excluded glucose and lactate because glucose concentrations below physiological levels accompanied by high lactate concentrations were detected in $64.4 \%$ of samples; this indicates glycolysis after blood collection, probably due to the time in post of whole blood before separating plasma from cell-containing blood fractions. Thus, 207 metabolite variables, including 75 ratios, were included in the statistical analysis.

Samples from 287 men were analysed at Nightingale Health, Helsinki, Finland (Figure S1). We excluded one participant (vegetarian), for whom $80.9 \%$ of metabolites had non-quantifiable values, leaving 286 men for the statistical analysis.

From three previous studies using split samples from the same baseline blood collection as for the NMR assay, we have MS-based plasma metabolite concentrations (AbsoluteIDQ ${ }^{\circledR}$ p180 Kit, Biocrates Life Sciences AG, Innsbruck, Austria) [20], standard clinical chemistry measurements of serum total cholesterol, non-HDL-cholesterol, HDL cholesterol, and apolipoproteins A-I and B [27], and GC measurements of plasma fatty acids DHA and LA [28], for some or all participants in the current study.

\subsection{Statistical Analysis}

Before analyses, all metabolite variables were logarithmically transformed to approximate the normal distribution. Pearson correlations between pairs of NMR metabolites were calculated.

To investigate the contribution of covariates (i.e., diet group, participant characteristics and factors related to blood collection and handling) to the variability in the NMR metabolite measures, we used the PC-PR2 method [32]. This method quantifies the proportion of the total variability in metabolite measures which is explained by each covariate after adjusting for all other covariates in the model.

Differences in geometric mean concentrations and ratios of metabolites across diet group were tested by ANOVA, adjusting for age (30-34, 35-39, 40-44, 45-49 years), BMI $\left(<22.5,22.5-24.9, \geq 25 \mathrm{~kg} / \mathrm{m}^{2}\right.$, unknown), smoking (never, former, current), alcohol intake $(<1,1-7,8-15, \geq 16 \mathrm{~g} / \mathrm{d})$, physical activity level (inactive, moderately inactive, moderately active, active, unknown), time since last meal at blood collection $(<1.5,1.5-2.9,3.0-4.4$, $\geq 4.5 \mathrm{~h}$, unknown) and time from blood collection to processing (quartiles of the distribution, i.e., $<24.5,24.6-41.3,41.4-71.6, \geq 71.7 \mathrm{~h}$, unknown). We controlled for multiple testing using the Benjamini-Hochberg false discovery rate (FDR) method with a significance level of 0.05 [52]. Due to the differences in units for the NMR metabolites, we repeated the analysis after $z$-score standardising (mean $=0$ and $\mathrm{SD}=1$ ) the log-transformed metabolite variables. In this analysis, we standardised to the adjusted mean in meat-eaters to provide an estimate of the difference from each of the non-meat-eating diet groups to the meat-eaters.

To explore latent patterns in metabolite profiles, and for dimension-reduction, we conducted multivariate analyses. For these analyses we excluded men with missing data on one or more of the 207 included metabolite measures $(n=49)$, leaving 237 men in the analyses. Overall z-score standardised log-transformed metabolite variables were used for these analyses.

First, we conducted a PCA, which maximises the variance explained in metabolite levels [53]. The relationship between the derived latent patterns in metabolite profile (principal components) and diet group was assessed using score plots.

Second, to obtain patterns in metabolite profile best differentiating diet groups, we ran PLS regression, which only accounts for metabolite information which is correlated with diet group [13] by maximising the covariance of the relationship between metabolite variables and diet group [53]. The number of components to retain was determined by assessing the goodness of fit $\left(R^{2} Y\right)$ and goodness of prediction $\left(Q^{2} Y\right)$, derived using sevenfold cross-validation; the algorithm identifies where the increase in $Q^{2} Y$ per additional 
component retained plateaus $[53,54]$. To assess the potential overfitting of the PLS models, we used permutations tests $(n=200)$, in which the assignment of diet group was random; higher goodness of fit and prediction for the actual model than the permutation models and negative values for goodness of prediction of the permutation models indicate validity of the actual model. We assessed which metabolites were most influential for the model using PLS weights for each retained component and VIP scores across all retained PLS components. The mean squared VIP is 1 and thus a VIP $>1$ is commonly used as a cut-off to denote important metabolites [53,54]. Two PLS models were run; one using all four diet groups and another including only meat-eaters and vegans $(n=112)$.

For metabolites measured using both NMR and one of the other methods, we compared the concentrations using Pearson correlations and Bland-Altman plots. Moreover, we calculated Pearson correlations between all metabolites measured using NMR and each of the other assay method.

We used R (Foundation for Statistical Computing, Vienna, Austria. URL: https: / / www.R-project.org / , accessed on 27 January 2021) for the statistical analyses of PC-PR2, PCA and PLS ('ropls' package for PCA and PLS). The remaining analyses were run using Stata Statistical Software Package, version 15 (Stata Corporation, College Station, TX, USA).

\section{Conclusions}

The metabolomics profiles of male meat-eaters, fish-eaters, vegetarians and especially vegans are different. This is mainly due to differences in fatty acids (lower levels of DHA, total n-3 fatty acids and SFA, and higher levels of LA, total n-6 fatty acids and PUFA in vegans compared to meat-eaters), and lower levels of cholesterol and triglycerides in VLDL, of various lipid fractions in HDL subclasses, and of sphingomyelins, tyrosine and creatinine, as well as higher alanine in vegans. Levels in fish-eaters and vegetarians differed by analyte but for many metabolite measures, the levels in fish-eaters and vegetarians were intermediate to those of meat-eaters and vegans.

There was moderate to strong comparability of the few specific metabolite concentrations measured both using NMR, and MS or clinical chemistry. Moreover, many strong correlations were found across all measured metabolites from NMR versus MS and especially clinical chemistry. Thus, the studied NMR and MS platforms are complementary.

Supplementary Materials: The following are available online at https:/ / www.mdpi.com/2218-198 9/11/2/121/s1, Figure S1. Exclusion of men and NMR metabolite measures, Figure S2. Cumulative goodness of fit (R2Y) and goodness of prediction $(\mathrm{Q} 2 \mathrm{Y})$ by the retained components (p1-p7) for the partial least squares model of 207 NMR metabolite measures in four diet groups, Figure S3. Validation of the partial least squares model of 207 NMR metabolite measures in four diet groups, Figure S4. Cumulative goodness of fit (R2Y) and goodness of prediction (Q2Y) by the retained components (p1-p6) for the partial least squares model of 207 NMR metabolite measures in meat-eaters and vegans, Figure S5. Validation of the partial least squares model of 207 NMR metabolite measures in meat-eaters and vegans, Figure S6. Bland-Altman plots comparing NMR metabolite concentrations to those measured using another method, Table S1. Pearson correlations between pairs of NMR metabolite measures in 286 men, Table S2. Geometric mean NMR metabolite measures by diet group on the original scale, Table S3. Geometric mean NMR metabolite measures by diet group standardised to the adjusted mean in meat-eaters, Table S4. Loadings from principal component analysis of four diet groups, Table S5. Weights and variable influence on projections (VIP) scores for the partial least squares models of 207 NMR metabolite measures in four diet groups and in meat-eaters and vegans, respectively, Table S6. Bland-Altman estimates of NMR metabolite concentrations compared to those measured using other methods, Table S7. Pearson correlations between NMR metabolite measures and metabolites measured using other methods in 286 men, Table S8. Completeness and coefficients of variation of metabolite measures from the NMR platform, and exclusion of metabolites for statistical analyses. 
Author Contributions: Conceptualization, J.A.S., M.V.H., T.J.K. and R.C.T.; data curation, J.A.S. and G.K.F.; formal analysis, J.A.S. and G.K.F.; funding acquisition, M.V.H., T.J.K. and R.C.T.; investigation, J.A.S., G.K.F., T.J.K. and R.C.T.; methodology, J.A.S., G.K.F., M.V.H., T.J.K. and R.C.T.; project administration, J.A.S. and R.C.T.; resources, S.R., A.S., M.J.G., T.J.K. and R.C.T.; software, J.A.S. and G.K.F.; supervision, T.J.K. and R.C.T.; visualization, J.A.S. and G.K.F.; writing-original draft preparation, J.A.S.; writing—review and editing, J.A.S., G.K.F., S.R., A.S., M.J.G., M.V.H., T.J.K. and R.C.T. All authors have read and agreed to the published version of the manuscript.

Funding: This research was funded by Cancer Research UK, grant numbers C8221/A19170 and C8221/29017 and the Nuffield Department of Population Health (NDPH) Pump Priming Scheme, Award Reference Number H6RD00410.

Institutional Review Board Statement: The study was conducted according to the guidelines of the Declaration of Helsinki, and the protocol for EPIC-Oxford was approved by a Multicentre Research Ethics Committee (MREC/02/0/90): Scotland A Research Ethics Committee (IRAS 223031; updated approval on 28/03/2018).

Informed Consent Statement: Informed consent was obtained from all subjects involved in the study.

Data Availability Statement: The data access policy for the EPIC-Oxford study is available via the study website (www.epic-oxford.org/data-access-sharing-and-collaboration/ (accessed on 20 February 2021)).

Acknowledgments: We thank all participants in the EPIC-Oxford cohort for their contribution to the study. We would also like to thank Paul Appleby for his help with data management, curation and analyses, Jakob Blaavand for technical help in producing the score and weight plots, and Frederik Romer for describing the technical details of the NMR assay.

Conflicts of Interest: The authors declare no conflict of interest. The funders had no role in the design of the study; in the collection, analyses, or interpretation of data; in the writing of the manuscript, or in the decision to publish the results.

Disclaimer: Where authors are identified as personnel of the International Agency for Research on Cancer/World Health Organization, the authors alone are responsible for the views expressed in this article and they do not necessarily represent the decisions, policy or views of the International Agency for Research on Cancer/World Health Organization.

\section{References}

1. Leitzmann, C. Vegetarian nutrition: Past, present, future. Am. J. Clin. Nutr. 2014, 100 (Suppl. S1), 496S-502S. [CrossRef]

2. Satija, A.; Bhupathiraju, S.N.; Spiegelman, D.; Chiuve, S.E.; Manson, J.E.; Willett, W.; Rexrode, K.M.; Rimm, E.B.; Hu, F.B. Healthful and Unhealthful Plant-Based Diets and the Risk of Coronary Heart Disease in U.S. Adults. J. Am. Coll. Cardiol. 2017, 70, 411-422. [CrossRef] [PubMed]

3. Kahleova, H.; Levin, S.; Barnard, N.D. Vegetarian Dietary Patterns and Cardiovascular Disease. Prog. Cardiovasc. Dis. 2018, 61, 54-61. [CrossRef]

4. Tong, T.Y.N.; Appleby, P.N.; Bradbury, K.E.; Perez-Cornago, A.; Travis, R.C.; Clarke, R.; Key, T.J. Risks of ischaemic heart disease and stroke in meat eaters, fish eaters, and vegetarians over 18 years of follow-up: Results from the prospective EPIC-Oxford study. BMJ 2019, 366, 14897. [CrossRef]

5. Orlich, M.J.; Singh, P.N.; Sabaté, J.; Fan, J.; Sveen, L.; Bennett, H.; Knutsen, S.F.; Beeson, W.L.; Jaceldo-Siegl, K.; Butler, T.L.; et al. Vegetarian Dietary Patterns and the Risk of Colorectal Cancers. JAMA Intern. Med. 2015, 175, 767-776. [CrossRef] [PubMed]

6. Key, T.J.; Appleby, P.N.; Crowe, F.L.; Bradbury, K.E.; Schmidt, J.A.; Travis, R.C. Cancer in British vegetarians: Updated analyses of 4998 incident cancers in a cohort of 32,491 meat eaters, 8612 fish eaters, 18,298 vegetarians, and 2246 vegans. Am. J. Clin. Nutr. 2014, 100 (Suppl. S1), 378S-385S. [CrossRef]

7. Tantamango-Bartley, Y.; Knutsen, S.F.; Knutsen, R.; Jacobsen, B.K.; Fan, J.; Beeson, W.L.; Sabate, J.; Hadley, D.; Jaceldo-Siegl, K.; Penniecook, J.; et al. Are strict vegetarians protected against prostate cancer? Am. J. Clin. Nutr. 2016, 103, 153-160. [CrossRef] [PubMed]

8. Tantamango-Bartley, Y.; Jaceldo-Siegl, K.; Fan, J.; Fraser, G. Vegetarian Diets and the Incidence of Cancer in a Low-risk Population. Cancer Epidemiol. Biomarkers Prev. 2013, 22, 286-294. [CrossRef]

9. Papier, K.; Appleby, P.N.; Fensom, G.K.; Knuppel, A.; Perez-Cornago, A.; Schmidt, J.A.; Tong, T.Y.N.; Key, T.J. Vegetarian diets and risk of hospitalisation or death with diabetes in British adults: Results from the EPIC-Oxford study. Nutr. Diabetes 2019,9 , 7. [CrossRef]

10. Tonstad, S.; Butler, T.; Yan, R.; Fraser, G.E. Type of Vegetarian Diet, Body Weight, and Prevalence of Type 2 Diabetes. Diabetes Care 2009, 32, 791-796. [CrossRef] [PubMed] 
11. Tong, T.Y.N.; Appleby, P.N.; Armstrong, M.E.G.; Fensom, G.K.; Knuppel, A.; Papier, K.; Perez-Cornago, A.; Travis, R.C.; Key, T.J. Vegetarian and vegan diets and risks of total and site-specific fractures: Results from the prospective EPIC-Oxford study. BMC Med. 2020, 18, 353. [CrossRef]

12. Scalbert, A.; Brennan, L.; Manach, C.; Andres-Lacueva, C.; Dragsted, L.O.; Draper, J.; Rappaport, S.M.; Van Der Hooft, J.J.J.; Wishart, D.S. The food metabolome: A window over dietary exposure. Am. J. Clin. Nutr. 2014, 99, 1286-1308. [CrossRef]

13. Guasch-Ferré, M.; Bhupathiraju, S.N.; Hu, F.B. Use of Metabolomics in Improving Assessment of Dietary Intake. Clin. Chem. 2018, 64, 82-98. [CrossRef]

14. Wurtz, P.; Havulinna, A.S.; Soininen, P.; Tynkkynen, T.; Prieto-Merino, D.; Tillin, T.; Ghorbani, A.; Artati, A.; Wang, Q.; Tiainen, M.; et al. Metabolite profiling and cardiovascular event risk: A prospective study of 3 population-based cohorts. Circulation 2015, 131, 774-785. [CrossRef]

15. Holmes, M.V.; Millwood, I.Y.; Kartsonaki, C.; Hill, M.R.; Bennett, D.A.; Boxall, R.; Guo, Y.; Xu, X.; Bian, Z.; Hu, R.; et al. Lipids, Lipoproteins, and Metabolites and Risk of Myocardial Infarction and Stroke. J. Am. Coll. Cardiol. 2018, 71, 620-632. [CrossRef]

16. Marklund, M.; Wu, J.H.; Imamura, F.; Del Gobbo, L.C.; Fretts, A.; De Goede, J.; Shi, P.; Tintle, N.; Wennberg, M.; Aslibekyan, S.; et al. Biomarkers of Dietary Omega-6 Fatty Acids and Incident Cardiovascular Disease and Mortality. Circulation 2019, 139, 2422-2436. [CrossRef]

17. Guasch-Ferré, M.; Hruby, A.; Toledo, E.; Clish, C.B.; Martínez-González, M.A.; Salas-Salvadó, J.; Hu, F.B. Metabolomics in Prediabetes and Diabetes: A Systematic Review and Meta-analysis. Diabetes Care 2016, 39, 833-846. [CrossRef]

18. His, M.; Viallon, V.; Dossus, L.; Gicquiau, A.; Achaintre, D.; Scalbert, A.; Ferrari, P.; Romieu, I.; Onland-Moret, N.C.; Weiderpass, E.; et al. Prospective analysis of circulating metabolites and breast cancer in EPIC. BMC Med. 2019, 17, 1-13. [CrossRef]

19. Schmidt, J.A.; Fensom, G.K.; Rinaldi, S.; Scalbert, A.; Appleby, P.N.; Achaintre, D.; Gicquiau, A.; Gunter, M.J.; Ferrari, P.; Kaaks, R.; et al. Patterns in metabolite profile are associated with risk of more aggressive prostate cancer: A prospective study of 3057 matched case-control sets from EPIC. Int. J. Cancer 2020, 146, 720-730. [CrossRef] [PubMed]

20. Schmidt, J.A.; Rinaldi, S.; Ferrari, P.; Carayol, M.; Achaintre, D.; Scalbert, A.; Cross, A.J.; Gunter, M.J.; Fensom, G.K.; Appleby, P.N.; et al. Metabolic profiles of male meat eaters, fish eaters, vegetarians, and vegans from the EPIC-Oxford cohort. Am. J. Clin. Nutr. 2015, 102, 1518-1526. [CrossRef]

21. Schmidt, J.A.; Rinaldi, S.; Scalbert, A.; Ferrari, P.; Achaintre, D.; Gunter, M.J.; Appleby, P.N.; Key, T.J.; Travis, R.C. Plasma concentrations and intakes of amino acids in male meat-eaters, fish-eaters, vegetarians and vegans: A cross-sectional analysis in the EPIC-Oxford cohort. Eur. J. Clin. Nutr. 2016, 70, 306-312. [CrossRef]

22. Wu, G.D.; Compher, C.; Chen, E.Z.; Smith, S.A.; Shah, R.D.; Bittinger, K.; Chehoud, C.; Albenberg, L.G.; Nessel, L.; Gilroy, E.; et al. Comparative metabolomics in vegans and omnivores reveal constraints on diet-dependent gut microbiota metabolite production. Gut 2016, 65, 63-72. [CrossRef]

23. Lindqvist, H.M.; Rådjursöga, M.; Malmodin, D.; Winkvist, A.; Ellegård, L. Serum metabolite profiles of habitual diet: Evaluation by ${ }^{1} \mathrm{H}$-nuclear magnetic resonance analysis. Am. J. Clin. Nutr. 2019, 110, 53-62. [CrossRef]

24. Wang, F.; Wan, Y.; Yin, K.; Wei, Y.; Wang, B.; Yu, X.; Ni, Y.; Zheng, J.; Huang, T.; Song, M.; et al. Lower circulating branched-chain amino acid concentrations among vegetarians are associated with changes in gut microbial composition and function. Mol. Nutr. Food Res. 2019, 63, e1900612. [CrossRef]

25. Xu, J.; Yang, S.; Cai, S.; Dong, J.; Li, X.; Chen, Z. Identification of biochemical changes in lactovegetarian urine using ${ }^{1} \mathrm{H}$ NMR spectroscopy and pattern recognition. Anal. Bioanal. Chem. 2010, 396, 1451-1463. [CrossRef]

26. Lindqvist, H.M.; Rådjursöga, M.; Torstensson, T.; Jansson, L.; Ellegård, L.; Winkvist, A. Urine Metabolite Profiles and Nutrient Intake Based on 4-Day Weighed Food Diary in Habitual Vegans, Vegetarians, and Omnivores. J. Nutr. 2021, 151, 30-39. [CrossRef] [PubMed]

27. Bradbury, K.E.; Crowe, F.L.; Appleby, P.N.; Schmidt, J.A.; Travis, R.C.; Key, T.J. Serum concentrations of cholesterol, apolipoprotein A-I and apolipoprotein B in a total of 1694 meat-eaters, fish-eaters, vegetarians and vegans. Eur. J. Clin. Nutr. 2014, 68, 178-183. [CrossRef]

28. Rosell, M.S.; Lloyd-Wright, Z.; Appleby, P.N.; Sanders, T.A.B.; Allen, N.E.; Key, T.J. Long-chain $n$-3 polyunsaturated fatty acids in plasma in British meat-eating, vegetarian, and vegan men. Am. J. Clin. Nutr. 2005, 82, 327-334. [CrossRef]

29. Yu, B.; Zanetti, K.A.; Temprosa, M.; Albanes, D.; Appel, N.; Barrera, C.B.; Ben-Shlomo, Y.; Boerwinkle, E.; Casas, J.P.; Clish, C.; et al. The Consortium of Metabolomics Studies (COMETS): Metabolomics in 47 Prospective Cohort Studies. Am. J. Epidemiol. 2019, 188, 991-1012. [CrossRef] [PubMed]

30. Rosell, M.; Appleby, P.; Spencer, E.; Key, T. Weight gain over 5 years in 21,966 meat-eating, fish-eating, vegetarian, and vegan men and women in EPIC-Oxford. Int. J. Obes. 2006, 30, 1389-1396. [CrossRef]

31. Perez-Cornago, A.; Huybrechts, I.; Appleby, P.N.; Schmidt, J.A.; Crowe, F.L.; Overvad, K.; Tjønneland, A.; Kühn, T.; Katzke, V.; Trichopoulou, A.; et al. Intake of individual fatty acids and risk of prostate cancer in the European Prospective Investigation into Cancer and Nutrition. Int. J. Cancer 2020, 146, 44-57. [CrossRef]

32. Fages, A.; Ferrari, P.; Monni, S.; Dossus, L.; Floegel, A.; Mode, N.; Johansson, M.; Travis, R.C.; Bamia, C.; Sánchez-Pérez, M.-J.; et al. Investigating sources of variability in metabolomic data in the EPIC study: The Principal Component Partial R-square (PC-PR2) method. Metabolomics 2014, 10, 1074-1083. [CrossRef] 
33. Pinto, A.M.; Sanders, T.A.B.; Kendall, A.C.; Nicolaou, A.; Gray, R.; Al-Khatib, H.; Hall, W.L. A comparison of heart rate variability, n-3 PUFA status and lipid mediator profile in age- and BMI-matched middle-aged vegans and omnivores. Br. J. Nutr. 2017, 117, 669-685. [CrossRef]

34. Dierckx, T.; Chiche, L.; Daniel, L.; Lauwerys, B.; Van Weyenbergh, J.; Jourde-Chiche, N. Serum GlycA Level is Elevated in Active Systemic Lupus Erythematosus and Correlates to Disease Activity and Lupus Nephritis Severity. J. Clin. Med. 2020, 9, 970. [CrossRef]

35. Tynkkynen, T.; Mursu, J.; Nurmi, T.; Tuppurainen, K.; Laatikainen, R.; Soininen, P. NMR protocol for determination of oxidation susceptibility of serum lipids and application of the protocol to a chocolate study. Metabolomics 2012, 8, 386-398. [CrossRef]

36. Suhre, K.; Meisinger, C.; Döring, A.; Altmaier, E.; Belcredi, P.; Gieger, C.; Chang, D.; Milburn, M.V.; Gall, W.E.; Weinberger, K.M.; et al. Metabolic footprint of diabetes: A multiplatform metabolomics study in an epidemiological setting. PLoS ONE 2010, 5, e13953. [CrossRef]

37. Raffler, J.; Römisch-Margl, W.; Petersen, A.-K.; Pagel, P.; Blöchl, F.; Hengstenberg, C.; Illig, T.; Meisinger, C.; Stark, K.; Wichmann, H.-E.; et al. Identification and MS-assisted interpretation of genetically influenced NMR signals in human plasma. Genome Med. 2013, 5, 13. [CrossRef]

38. Yet, I.; Menni, C.; Shin, S.-Y.; Mangino, M.; Soranzo, N.; Adamski, J.; Suhre, K.; Spector, T.D.; Kastenmüller, G.; Bell, J.T. Genetic Influences on Metabolite Levels: A Comparison across Metabolomic Platforms. PLoS ONE 2016, 11, e0153672. [CrossRef]

39. Wu, J.H.Y.; Marklund, M.; Imamura, F.; Tintle, N.; Korat, A.V.A.; De Goede, J.; Zhou, X.; Yang, W.-S.; de Oliveira Otto, M.C.; Kröger, J.; et al. Omega-6 fatty acid biomarkers and incident type 2 diabetes: Pooled analysis of individual-level data for 39,740 adults from 20 prospective cohort studies. Lancet Diabetes Endocrinol. 2017, 5, 965-974. [CrossRef]

40. Imamura, F.; Sharp, S.J.; Koulman, A.; Schulze, M.B.; Kröger, J.; Griffin, J.L.; Huerta, J.M.; Guevara, M.; Sluijs, I.; Agudo, A.; et al. A combination of plasma phospholipid fatty acids and its association with incidence of type 2 diabetes: The EPIC-InterAct case-cohort study. PLoS Med. 2017, 14, e1002409. [CrossRef]

41. Stevens, V.L.; Hoover, E.; Wang, Y.; Zanetti, K.A. Pre-Analytical Factors that Affect Metabolite Stability in Human Urine, Plasma, and Serum: A Review. Metabolites 2019, 9, 156. [CrossRef]

42. Santos Ferreira, D.L.; Maple, H.J.; Goodwin, M.; Brand, J.S.; Yip, V.; Min, J.L.; Groom, A.; Lawlor, D.A.; Ring, S. The Effect of Pre-Analytical Conditions on Blood Metabolomics in Epidemiological Studies. Metabolites 2019, 9, 64. [CrossRef]

43. Wurtz, P.; Wang, Q.; Soininen, P.; Kangas, A.J.; Fatemifar, G.; Tynkkynen, T.; Tiainen, M.; Perola, M.; Tillin, T.; Hughes, A.D.; et al. Metabolomic Profiling of Statin Use and Genetic Inhibition of HMG-CoA Reductase. J. Am. Coll. Cardiol. 2016, 67, 1200-1210. [CrossRef] [PubMed]

44. Kaluarachchi, M.; Boulangé, C.L.; Karaman, I.; Lindon, J.C.; Ebbels, T.M.D.; Elliott, P.; Tracy, R.P.; Olson, N.C. A comparison of human serum and plasma metabolites using untargeted ${ }^{1} \mathrm{H}$ NMR spectroscopy and UPLC-MS. Metabolomics 2018, 14, 1-12. [CrossRef]

45. Li-Gao, R.; Hughes, D.A.; Le Cessie, S.; De Mutsert, R.; Heijer, M.D.; Rosendaal, F.R.; Van Dijk, K.W.; Timpson, N.J.; MookKanamori, D.O. Assessment of reproducibility and biological variability of fasting and postprandial plasma metabolite concentrations using ${ }^{1} \mathrm{H}$ NMR spectroscopy. PLoS ONE 2019, 14, e0218549. [CrossRef] [PubMed]

46. Davey, G.K.; Spencer, E.A.; Appleby, P.N.; Allen, N.E.; Knox, K.H.; Key, T.J. EPIC-Oxford: Lifestyle characteristics and nutrient intakes in a cohort of 33,883 meat-eaters and 31,546 non meat-eaters in the UK. Public Health Nutr. 2003, 6, 259-268. [CrossRef]

47. Bingham, S.A.; Cassidy, A.; Cole, T.J.; Welch, A.; Runswick, S.A.; Black, A.E.; Thurnham, D.; Bates, C.; Khaw, K.T.; Key, T.J.A.; et al. Validation of weighed records and other methods of dietary assessment using the $24 \mathrm{~h}$ urine nitrogen technique and other biological markers. Br. J. Nutr. 1995, 73, 531-550. [CrossRef]

48. Bingham, S.A.; Gill, C.; Welch, A.; Day, K.; Cassidy, A.; Khaw, K.T.; Sneyd, M.J.; Key, T.J.A.; Roe, L.; Day, N.E. Comparison of dietary assessment methods in nutritional epidemiology: Weighed records v. $24 \mathrm{~h}$ recalls, food-frequency questionnaires and estimated-diet records. Br. J. Nutr. 1994, 72, 619-643. [CrossRef]

49. Soininen, P.; Kangas, A.J.; Raitakari, O.T.; Savolainen, M.J.; Ala-Korpela, M.; Würtz, P.; Tukiainen, T.; Tynkkynen, T.; Laatikainen, R.; Järvelin, M.-R.; et al. High-throughput serum NMR metabonomics for cost-effective holistic studies on systemic metabolism. Analyst 2009, 134, 1781-1785. [CrossRef] [PubMed]

50. Soininen, P.; Kangas, A.J.; Würtz, P.; Suna, T.; Ala-Korpela, M. Quantitative Serum Nuclear Magnetic Resonance Metabolomics in Cardiovascular Epidemiology and Genetics. Circ. Cardiovasc. Genet. 2015, 8, 192-206. [CrossRef] [PubMed]

51. Würtz, P.; Kangas, A.J.; Soininen, P.; Lawlor, D.A.; Smith, G.D.; Ala-Korpela, M. Quantitative Serum Nuclear Magnetic Resonance Metabolomics in Large-Scale Epidemiology: A Primer on -Omic Technologies. Am. J. Epidemiol. 2017, 186, 1084-1096. [CrossRef] [PubMed]

52. Benjamini, Y.; Hochberg, Y. Controlling the False Discovery Rate-A Practical and Powerful Approach to Multiple Testing. J. R. Stat. Soc. Ser. B Methodol. 1995, 57, 289-300. [CrossRef]

53. Eriksson, L.; Byrne, T.; Johansson, E.; Trygg, J.; Vikström, C. Multi-and Megavariate Data Analysis Basic-Principles and Applications, 3rd ed.; MKS Umetrics AB: Malmö, Sweden, 2013; pp. 55-88.

54. Simeone, P.; Trerotola, M.; Urbanella, A.; Lattanzio, R.; Ciavardelli, D.; Di Giuseppe, F.; Eleuterio, E.; Sulpizio, M.; Eusebi, V.; Pession, A.; et al. A Unique Four-Hub Protein Cluster Associates to Glioblastoma Progression. PLoS ONE 2014, 9, e103030. [CrossRef] [PubMed] 